\title{
LA VEGETACIÓN DE LAGUNAS PRIMAVERALES EN LAS CERCANÍAS DE TEMUCO (CAUTÍN, CHILE)
}

\author{
Cristina SAN MARTÍN PADOVANI, Carlos RAMÍREZ GARCÍA y Pedro OJEDA NEMPU
}

\begin{abstract}
RESUMEN. La vegetación de lagunas primaverales en las cercanías de Temuco (Cautín, Chile). Se estudió la vegetación de lagunas primaverales ubicadas en las serranías de Tromén al poniente de la ciudad de Temuco, Chile. El estudio se realizó usando la metodología fitosociológica tradicional y modernos métodos estadísticos multivariantes de clasificación y ordenación. Se determinaron las siguientes asociaciones: Juncetum procerii, Mentho-Agrostietum capillaris, Eleocharitetum pachycarpae, Eleocharitetum macrostachyae, Gnaphalio-Polygonetum hydropiperoidis, Glycerietum multiflorae y Junco-Caricetum ripariae y las comunidades vegetales de Ludwigia peploides-Sagittaria montevidense, Eleocharis pachycarpaLythrum portula, Anthoxanthum utriculatum, Phyla nodiflora y Potamogeton pusillus. Estas asociaciones y comunidades se compararon en su distribución, en el origen de su flora y en sus espectros biológicos. La clasificación estadística coincidió con aquella realizada por el ordenamiento tradicional de la tabla fitosociológica inicial. La ordenación multivariante permitió inferir que la humedad del suelo, la extensión del período de sequía estival y la materia orgánica del sustrato son los principales factores que regulan la distribución de las especies y asociaciones vegetales.
\end{abstract}

Palabras clave. Humedales, vegetación hidrófila, fitosociología, clasificación, ordenación, Araucanía, Chile.

ABSTRACT. The vegetation of the vernal pools near Temuco (Cautin, Chile). The vegetation of the vernal pools located in Tromen Hill near Temuco city (Chile) was studied. The study was made using the tradicional plantsociological methods and with modern statistical multivariate methods of classification and ordination. The following plant associations were determined: Juncetum procerii, Mentho-Agrostietum capillaris, Eleocharitetum pachycarpae, Eleocharitetum macrostachyae, Gnaphalio-Polygonetum hydropiperoidis, Glycerietum multiflorae and Junco-Caricetum ripariae and so the communities of Ludwigia peploides-Sagittaria montevidense, Eleocharis pachycarpa-Lythrum portula, Anthoxanthum utriculatum, Phyla nodiflora and Potamogeton pusillus. These plant associations and communities were compared in his distribution, origin of the flora and biological spectra. The statistical classification was similar to the traditional ordination of the phytosociological table. The multivariate ordination allowed to infer that the wet conditions, the extension of the dry summer period and the organic matter of the substrat are the most important factors in the distribution of species and plant associations.

Key words. Wetlands, hydrophylic vegetation, phytosociology, classification, ordination, Araucanía, Chile.

Trabajo realizado con cargo a los proyectos $\mathrm{N}^{\circ} \mathrm{TS} 3 * \mathrm{CT} 94-03535$ de la Comunidad Europea y al $\mathrm{N}^{\circ}$ : F-95-06 de la Dirección de Investigación y Desarrollo de la Universidad Austral de Chile con . 


\section{INTRODUCCIÓN}

Las serranías de Tromén, ubicadas en la cordillera costera, al poniente de la ciudad de Temuco (Chile) estuvieron originalmente cubiertas por un bosque templado caducifolio, perteneciente a la asociación NothofagoPerseetum linguae (Bosque de Roble-LaurelLingue) y a la subasociación de la misma, Nothofago-Perseetum linguae boldetosum, con elementos esclerófilos en los estratos arbóreos medio y bajo (Ramírez et al., 1989, 1997, San Martín et al., 1991). En depresiones cerradas y junto a pequeños cursos de agua, el bosque original de tipo pantanoso pertenecía a la asociación Blepharocalyo-Myrceugenietum exsuccae (Bosque Temo-Pitra) (Ramírez et al., 1996).

En la actualidad, estas serranías albergan a reducciones indígenas, cuyas necesidades de subsistencia las han llevado a destruir dichos bosques (caducifolios y pantanosos) para obtener tierras de cultivo y de pastoreo, respectivamente (Ramírez et al., 1989). En las pequeñas depresiones cerradas y pastoreadas, se formaron así, lagunas estacionales que reducen su contenido de agua en primavera y se secan totalmente en verano (San Martín et $a l ., 1997)$. En estas épocas del año se produce primero un rápido crecimiento de la vegetación litoral perenne, pratense y pantanosa y más tarde, la aparición en el centro de las lagunas de una vegetación efímera de prados anuales, que cumplen su ciclo de vida en la época de sequía.

Se estudia la diversidad vegetacional de estas lagunas primaverales y la disposición de sus asociaciones en la zonación litoral, suponiendo que deberían ser muy semejantes en todas ellas.

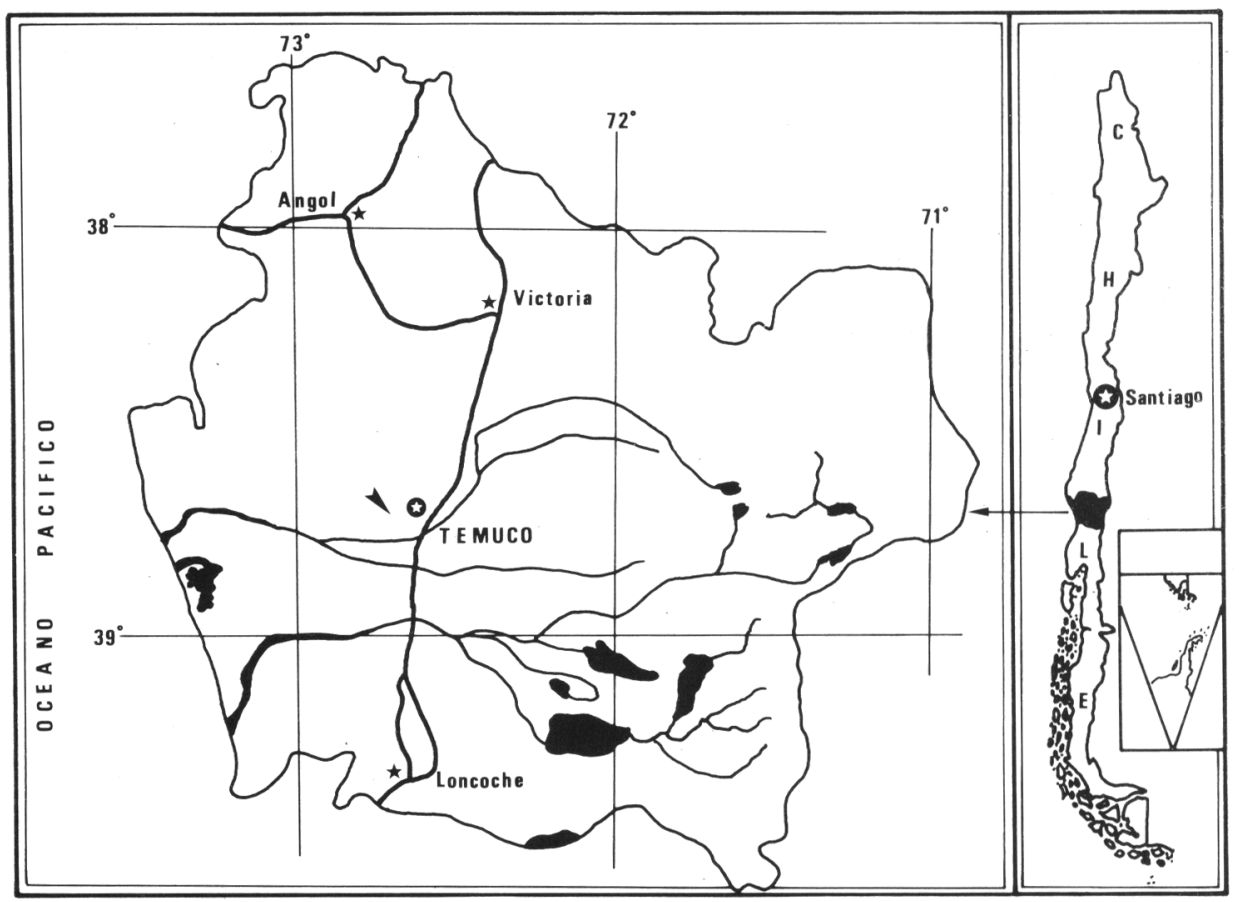

Figura 1. Novena región de Chile. La punta de flecha señala el lugar de trabajo en las cercanías de Temuco. Ninth region of Chile. The point arrow indicates the work site near Temuco. 


\section{MATERIALES Y MÉTODOS}

\section{Área de estudio}

El estudio se realizó en 10 lagunas efímeras primaverales ubicadas en las serranías de Tromén, cerca de la ciudad de Temuco (fig. 1). El suelo del lugar es del tipo rojo arcilloso perteneciente en su mayoría a la serie Metrenco (Besoaín, 1985) el que por su alto contenido en arcillas es pesado y difícil de labrar, quedando restringido su laboreó a un par de semanas en el año.

El clima de la ciudad de Temuco es del tipo templado lluvioso con influencia mediterránea (Novoa y Villaseca, 1989). La temperatura promedio anual alcanza a $12^{\circ} \mathrm{C}$ y la precipitación anual a $1325 \mathrm{~mm}$ (Hajek y Di Castri, 1975). Aunque las lluvias se concentran en la época invernal, existen a los menos, dos meses secos en el período estival(Di Castri y Hajek, 1976). Este período que en años secos puede extenderse por varios meses, condiciona la flora y la vegetación de las lagunas primaverales estudiadas.

Las lagunas investigadas son la única fuente de agua potable para humanos y ganado en épocas de sequía, por ello todas muestran una gran influencia antrópica. Esta influencia se refleja por un lado en la eutrofización del sustrato y del agua y por otro, en la compactación del suelo por pisoteo. Además, la erosión de las colinas adyacentes aumenta la sedimentación en las lagunas, disminuyendo la profundidad y consecuentemente, aumentando la duración del período de sequía estival. Por último, en el borde de ellas se observa degradación de los suelos por cultivos reiterados.

\section{Material}

La base del estudio vegetacional de las lagunas la constituyó una tabla fitosociológica, confeccionada con 86 censos de vegetación, levantados con la metodología sigmatista desarrollada por la Escuela europea ZürichMontpellier de fitosociología (Dierschcke, 1994).

Los censos de vegetación se levantaron en lugares florística-, fisonómica- y ecológicamente homogéneos tratando de abarcar con ellos, toda la diversidad vegetacional observada a simple vista (Knapp, 1984). Cada censo se realizó en una superficie de $25 \mathrm{~m} 2(5 \times 5 \mathrm{~m})$, suficiente para captar la estructura y composición florística de comunidades herbáceas, pratenses y pantanosas (Nuñez, 1987). En cada censo se confeccionó una lista exhaustiva de las especies presentes y luego se estimó la abundancia de los individuos de cada una de ellas, expresándola en porcentaje de cobertura de la parcela (Knapp, 1984). Para coberturas inferiores al $1 \%$ se usaron los signos «+» $\mathrm{y}$ «r» (cruz y erre); el primero cuando habían varios individuos de la especie en cuestión y el segundo, cuando sólo se presentaba uno (Ellenberg, 1956). La distribución de las lagunas muestreadas se representa en la figura 2 .

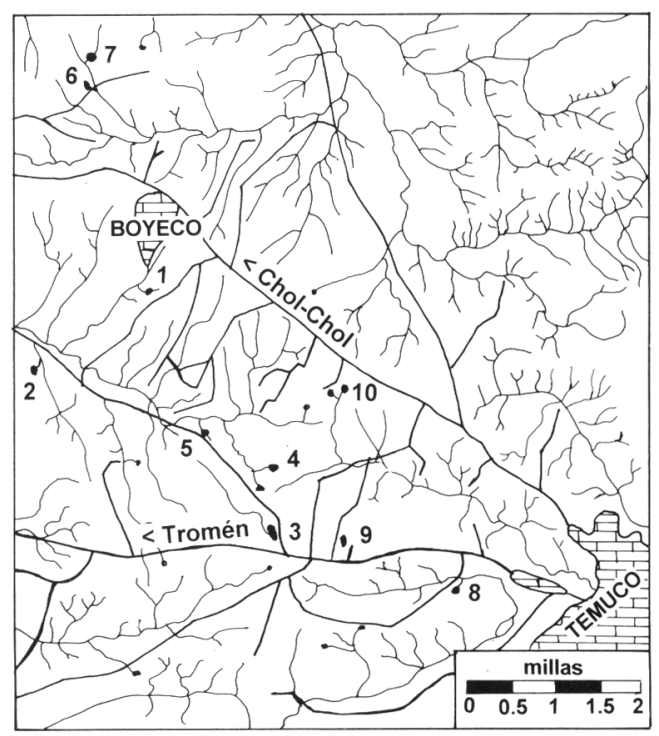

Figura 2. Ubicación de las lagunas estudiadas. Location of the studied lagoons. 


\begin{tabular}{|c|c|c|c|c|c|c|c|c|c|c|c|c|}
\hline Especies / Asociaciones: & $\mathrm{Jp}$ & MA & Ep & $\mathrm{Em}$ & GP & LS & LE & $\mathrm{Au}$ & Pn & $\mathrm{Gm}$ & $\mathrm{Cr}$ & Pp \\
\hline Mentha pulegium & 07 & 47 & 10 & 12 & 04 & 00 & 01 & 01 & 01 & 00 & 01 & 00 \\
\hline Agrostis capillaris & 21 & 06 & 01 & 01 & 00 & 00 & 00 & 03 & 00 & 01 & 10 & 00 \\
\hline Polygonum hydropiperoides & 02 & 03 & 03 & 01 & 20 & 00 & 01 & 00 & 00 & 00 & 01 & 00 \\
\hline Leontodon saxatile & 05 & 12 & 01 & 01 & 01 & 00 & 00 & 20 & 01 & 00 & 00 & 00 \\
\hline Juncus procerus & 55 & 01 & 01 & 00 & 00 & 00 & 01 & 15 & 00 & 00 & 05 & 00 \\
\hline Eleocharis pachycarpa & 03 & 01 & 62 & 01 & 01 & 00 & 17 & 00 & 00 & 00 & 00 & 00 \\
\hline Eleocharis macrostachya & 01 & 01 & 01 & 65 & 01 & 00 & 00 & 00 & 03 & 00 & 00 & 00 \\
\hline Phyla nodiflora & 01 & 01 & 02 & 04 & 01 & 00 & 00 & 00 & 95 & 00 & 00 & 00 \\
\hline Rumex conglomeratus & 01 & 01 & 01 & 01 & 00 & 00 & 00 & 00 & 01 & 00 & 01 & 00 \\
\hline Cyperus reflexus & 01 & 01 & 01 & 01 & 01 & 00 & 00 & 00 & 01 & 00 & 00 & 00 \\
\hline Gratiola peruviana & 01 & 01 & 07 & 03 & 01 & 01 & 00 & 00 & 00 & 00 & 00 & 0 \\
\hline Centipeda elatinoides & 01 & 01 & 01 & 03 & 03 & 00 & 01 & 00 & 00 & 00 & 00 & 00 \\
\hline Rumex acetosella & 01 & 01 & 00 & 01 & 01 & 00 & 00 & 01 & 01 & 00 & 00 & 00 \\
\hline Glyceria multiflora & 01 & 01 & 01 & 01 & 00 & 00 & 00 & 00 & 00 & 25 & 00 & 00 \\
\hline Gnaphalium cymatoides & 00 & 01 & 01 & 04 & 27 & 00 & 05 & 00 & 00 & 00 & 00 & 00 \\
\hline Lotus uliginosus & 06 & 08 & 01 & 01 & 00 & 00 & 00 & 01 & 00 & 00 & 00 & 00 \\
\hline Lythrum portula & 00 & 00 & 01 & 00 & 00 & 01 & 72 & 00 & 00 & 01 & 00 & 00 \\
\hline Juncus microcephalus & 01 & 03 & 01 & 01 & 00 & 00 & 00 & 00 & 00 & 00 & 00 & 00 \\
\hline Eryngium pseudojunceum & 01 & 01 & 01 & 02 & 00 & 00 & 00 & 00 & 00 & 00 & 00 & 00 \\
\hline Juncus imbricatus & 04 & 01 & 01 & 00 & 00 & 00 & 00 & 01 & 00 & 00 & 00 & 00 \\
\hline Plantago lanceolata & 01 & 01 & 01 & 00 & 01 & 00 & 00 & 00 & 00 & 00 & 00 & 00 \\
\hline Spergularia rubra & 00 & 01 & 01 & 01 & 03 & 00 & 00 & 00 & 00 & 00 & 00 & 00 \\
\hline Setaria parvifolia & 01 & 01 & 01 & 00 & 00 & 00 & 00 & 00 & 01 & 00 & 00 & 00 \\
\hline ia peploides & 01 & 00 & 00 & 00 & 00 & 04 & 00 & 00 & 00 & 10 & 00 & 00 \\
\hline dicata & 01 & 00 & 01 & 00 & 00 & 00 & 00 & 01 & 00 & 00 & 00 & 00 \\
\hline Holcus lanatus & 02 & 01 & 00 & 00 & 00 & 00 & 00 & 01 & 00 & 00 & 00 & 00 \\
\hline Dichondra sericea & 01 & 01 & 00 & 00 & 00 & 00 & 00 & 01 & 00 & 00 & 00 & 00 \\
\hline Chamomilla recutita & 01 & 00 & 00 & 00 & 01 & 00 & 00 & 00 & 01 & 00 & 00 & 00 \\
\hline aeta americana & 01 & 01 & 00 & 00 & 00 & 00 & 00 & 01 & 00 & 00 & 00 & 00 \\
\hline Poly & 01 & 01 & 01 & 00 & 00 & 00 & 00 & 00 & 00 & 00 & 00 & 00 \\
\hline chantioides & 01 & 01 & 00 & 00 & 00 & 00 & 00 & 05 & 00 & 00 & 00 & 00 \\
\hline Prunella vulgaris & 01 & 01 & 00 & 00 & 00 & 00 & 00 & 01 & 00 & 00 & 00 & 00 \\
\hline Callitriche terrestris & 01 & 00 & 00 & 00 & 00 & 01 & 01 & 00 & 00 & 00 & 00 & 00 \\
\hline Cyperus eragrostis & 01 & 00 & 01 & 00 & 00 & 00 & 01 & 00 & 00 & 00 & 00 & 00 \\
\hline Oxalis corniculata & 00 & 01 & 01 & 00 & 03 & 00 & 00 & 00 & 00 & 00 & 00 & 00 \\
\hline lyg & 00 & 00 & 01 & 00 & 01 & 00 & 01 & 00 & 00 & 00 & 00 & 00 \\
\hline volucrata & 00 & 01 & 00 & 05 & 01 & 00 & 00 & 00 & 00 & 00 & 0 & 00 \\
\hline Glyn & 00 & 00 & 00 & 01 & 01 & 00 & 01 & 00 & 00 & 00 & 00 & 00 \\
\hline Sagittaria montevidense & 00 & 00 & 00 & 00 & 00 & 59 & 00 & 00 & 00 & 20 & 00 & 00 \\
\hline nthum utric & 01 & 00 & 00 & 00 & 00 & 00 & 00 & 55 & 00 & 00 & 00 & 00 \\
\hline Eryngium humiffusum & 00 & 03 & 00 & 01 & 00 & 00 & 00 & 00 & 00 & 00 & 00 & 00 \\
\hline Aster vahlii & 01 & 01 & 00 & 00 & 00 & 00 & 00 & 00 & 00 & 00 & 00 & 00 \\
\hline Rubus constrictl & 01 & 00 & 00 & 00 & 00 & 00 & 00 & 01 & 00 & 00 & 00 & 00 \\
\hline Myriophyllum aquaticum & 01 & 00 & 00 & 00 & 00 & 01 & 00 & 00 & 00 & 00 & 00 & 00 \\
\hline Hedyotis salzr & 01 & 00 & 00 & 00 & 00 & 00 & 00 & 01 & 00 & 00 & 00 & 00 \\
\hline Callitriche autumnalis & 00 & 00 & 00 & 00 & 00 & 01 & 00 & 00 & 00 & 01 & 00 & 00 \\
\hline Cyperus conceptionis & 01 & 01 & 00 & 00 & 00 & 00 & 00 & 00 & 00 & 00 & 00 & 00 \\
\hline Cuscuta suaveolens & 01 & 01 & 00 & 00 & 00 & 00 & 00 & 00 & 00 & 00 & 00 & 00 \\
\hline Gamochaeta spiciforme & 01 & 00 & 00 & 01 & 00 & 00 & 00 & 00 & 00 & 00 & 00 & 00 \\
\hline Alisma plantago-aquatica & 00 & 00 & 01 & 00 & 00 & 00 & 01 & 00 & 00 & 00 & 00 & 00 \\
\hline Myosotis arvensis & 00 & 00 & 01 & 00 & 00 & 00 & 01 & 00 & 00 & 00 & 00 & 00 \\
\hline Crassula peduncularis & 00 & 00 & 01 & 00 & 00 & 00 & 01 & 00 & 00 & 00 & 00 & 00 \\
\hline Carex & 00 & 00 & 00 & 00 & 00 & 00 & 00 & 00 & 00 & 00 & 90 & 00 \\
\hline Potamogeton pusillus & 00 & 00 & 00 & 00 & 00 & 00 & 00 & 00 & 00 & 00 & 00 & 99 \\
\hline Nothoscordur & 00 & 01 & 00 & 00 & 00 & 00 & 00 & 00 & 00 & 00 & 00 & 00 \\
\hline
\end{tabular}




\begin{tabular}{|c|c|c|c|c|c|c|c|c|c|c|c|c|}
\hline Especies / Asociaciones: & $\mathrm{Jp}$ & MA & Ep & $\mathrm{Em}$ & GP & LS & LE & $\mathrm{Au}$ & $\operatorname{Pn}$ & $\mathrm{Gm}$ & $\mathrm{Cr}$ & $\mathrm{Pp}$ \\
\hline Daucus carota & 01 & 00 & 00 & 00 & 00 & 00 & 00 & 00 & 00 & 00 & 00 & 00 \\
\hline Piptochaetium montevidense & 01 & 00 & 00 & 00 & 00 & 00 & 00 & 00 & 00 & 00 & 00 & 00 \\
\hline Juncus stipulatus & 01 & 00 & 00 & 00 & 00 & 00 & 00 & 00 & 00 & 00 & 00 & 00 \\
\hline Briza minor & 00 & 01 & 00 & 00 & 00 & 00 & 00 & 00 & 00 & 00 & 00 & 00 \\
\hline Parentucellia viscosa & 00 & 01 & 00 & 00 & 00 & 00 & 00 & 00 & 00 & 00 & 00 & 00 \\
\hline Lotus corniculatus & 01 & 00 & 00 & 00 & 00 & 00 & 00 & 00 & 00 & 00 & 00 & 00 \\
\hline Scirpus californicus & 00 & 00 & 00 & 00 & 00 & 10 & 00 & 00 & 00 & 00 & 00 & 00 \\
\hline Utricularia gibba & 00 & 00 & 00 & 00 & 00 & 01 & 00 & 00 & 00 & 00 & 00 & 00 \\
\hline Limosella australis & 00 & 00 & 00 & 00 & 00 & 01 & 00 & 00 & 00 & 00 & 00 & 00 \\
\hline Myrceugenia exsucca & 01 & 00 & 00 & 00 & 00 & 00 & 00 & 00 & 00 & 00 & 00 & 00 \\
\hline Arrhenatherum elatius & 01 & 00 & 00 & 00 & 00 & 00 & 00 & 00 & 00 & 00 & 00 & 00 \\
\hline Muehlenbeckia hastulata & 00 & 01 & 00 & 00 & 00 & 00 & 00 & 00 & 00 & 00 & 00 & 00 \\
\hline Vulpia bromoides & 00 & 01 & 00 & 00 & 00 & 00 & 00 & 00 & 00 & 00 & 00 & 00 \\
\hline Juncus bufonius & 00 & 00 & 00 & 00 & 01 & 00 & 00 & 00 & 00 & 00 & 00 & 00 \\
\hline Cirsium vulgare & 00 & 02 & 00 & 00 & 00 & 00 & 00 & 00 & 00 & 00 & 00 & 00 \\
\hline Cynosurus echinatus & 00 & 01 & 00 & 00 & 00 & 00 & 00 & 00 & 00 & 00 & 00 & 00 \\
\hline Achillea millefolium & 00 & 01 & 00 & 00 & 00 & 00 & 00 & 00 & 00 & 00 & 00 & 00 \\
\hline Modiola caroliniana & 00 & 01 & 00 & 00 & 00 & 00 & 00 & 00 & 00 & 00 & 00 & 00 \\
\hline Polygonum aviculare & 00 & 00 & 00 & 00 & 00 & 00 & 00 & 00 & 01 & 00 & 00 & 00 \\
\hline Crepis capillaris & 00 & 00 & 00 & 00 & 00 & 00 & 00 & 01 & 00 & 00 & 00 & 00 \\
\hline Sysirinchium graminifolium & 00 & 01 & 00 & 00 & 00 & 00 & 00 & 00 & 00 & 00 & 00 & 00 \\
\hline Geranium robertianum & 00 & 00 & 00 & 00 & 00 & 00 & 00 & 01 & 00 & 00 & 00 & 00 \\
\hline Trifolium repens & 00 & 00 & 00 & 00 & 00 & 00 & 00 & 01 & 00 & 00 & 00 & 00 \\
\hline Paspalum distichum & 00 & 00 & 00 & 00 & 00 & 00 & 00 & 01 & 00 & 00 & 00 & 00 \\
\hline Baccharis sagittalis & 01 & 00 & 00 & 00 & 00 & 00 & 00 & 00 & 00 & 00 & 00 & 00 \\
\hline Echinochloa crusgalli & 00 & 00 & 01 & 00 & 00 & 00 & 00 & 00 & 00 & 00 & 00 & 00 \\
\hline Panicum capillare & 00 & 00 & 01 & 00 & 00 & 00 & 00 & 00 & 00 & 00 & 00 & 00 \\
\hline Veronica anagallis-aquatica & 00 & 00 & 00 & 00 & 00 & 00 & 01 & 00 & 00 & 00 & 00 & 00 \\
\hline Juncus dombeỹanus & 00 & 00 & 00 & 00 & 00 & 00 & 01 & 00 & 00 & 00 & 00 & 00 \\
\hline Ranunculus bonariensis & 00 & 00 & 01 & 00 & 00 & 00 & 00 & 00 & 00 & 00 & 00 & 00 \\
\hline Rorippa palustris & 00 & 00 & 01 & 00 & 00 & 00 & 00 & 00 & 00 & 00 & 00 & 00 \\
\hline
\end{tabular}

Asociaciones o Comunidades: $\mathrm{Jp}=$ Juncetum procerii, $\mathrm{MA}=$ Mentho-Agrostietum capillaris, $\mathrm{Ep}=$ Eleocharitetum pachycarpae, $\mathrm{Em}=$ Eleocharitetum macrostachyae, $\mathrm{GP}=$ Gnaphalio-Polygonetum hydropiperoidis, $\mathrm{LS}=$ Ludwigia peploides-Sagittaria montevidense, LE = Eleocharis pachycarpa-Lythrum portula, $\mathrm{Au}=$ de Anthoxanthum utriculatum, $\mathrm{Pn}=$ de Phyla nodiflora, $\mathrm{Gm}=$ Glycerietum multiflorae, $\mathrm{Cr}$ = Junco-Caricetum ripariae, $\mathrm{Pp}=$ de Potamogeton pusillus.

Tabla 1. Matriz de datos usada para el análisis estadístico multivariado. Los valores indican porcentaje de cobertura promedio. Data matrix used for the multivariante statistical analysis. Value data indicate average cover percentage.

\section{Método}

Con los 86 censos se preparó una tabla fitosociológica inicial que incluyó las 86 especies vegetales presentes. La flora de esta tabla fue analizada en detalle por San Martín et al. (1997). Para este trabajo, la tabla fitosociológica inicial fue ordenada en forma tradicional con la metodología descrita por
Ramírez y Westermeier (1976), usando especies diferenciales que se excluían mutuamente (Kreeb, 1983). En esta tabla se diferenciaron sintaxa, que se describen y nombran de acuerdo a las normas internacionales de nomenclatura fitosociológica (Barkmann et al., 1986). Para cada sintaxón determinado se estructuró una tabla fitosociológica parcial en la cual se analizaron los espectros de origen y biológico. 
Posteriormente, con el promedio de cobertura de cada tabla parcial se confeccionó una tabla resumida que sirvió como matriz de datos con 12 casos (asociaciones) y 86 variables (especies). En esta matriz los signos «+»y «r» (cruz y erre) fueron elevados a la unidad (tab. 1). Ella fue sometida a análisis estadísticos multivariados de clasificación y ordenación (Orloci, 1975; Digby y Kempton, 1987). Para el análisis de conglomerados se usó el coeficiente de correlación de Pearson (Sáiz, 1980) y el dendrograma se construyó mediante la técnica del salto mínimo (Gauch, 1975).

\section{RESULTADOS Y DISCUSIÓN}

Mediante la selección de 19 especies diferenciales se ordenó la tabla fitosociológica preparada con los censos de vegetación, lográndose determinar 12 asociaciones vegetales, lo que representa una alta diversidad vegetacional, que está indicando una alta heterogeneidad de hábitats y la presencia de diferentes biótopos en las lagunas. De estas comunidades, 5 ya habían sido descritas en la literatura científica y las 7 restantes, corresponden a asociaciones vegetales nuevas. Se describen a continuación, las comunidades vegetales determinadas:

1. Pradera húmeda de Junquillo

Juncetum procerii Oberdorfer 1960

(tab. 2)

Oberdorfer 1960. Pflanzensoziologische Studien in Chile: Ein Vergleich mit Europe. Flora et Vegetatio Mundi 2: 1 - 208.

Se trata de una comunidad pratense densa

\begin{tabular}{|c|c|c|c|c|c|c|c|c|c|c|c|c|c|c|c|}
\hline $\begin{array}{l}\text { Laguna: } \\
\text { Especies / Censos: }\end{array}$ & $\begin{array}{l}1 \\
1\end{array}$ & $\begin{array}{l}1 \\
2\end{array}$ & $\begin{array}{l}2 \\
3\end{array}$ & $\begin{array}{l}2 \\
4\end{array}$ & $\begin{array}{l}2 \\
5\end{array}$ & $\begin{array}{l}2 \\
6\end{array}$ & $\begin{array}{l}4 \\
7\end{array}$ & $\begin{array}{l}6 \\
8\end{array}$ & $\begin{array}{c}10 \\
9\end{array}$ & $\begin{array}{c}2 \\
10\end{array}$ & $\begin{array}{c}3 \\
11\end{array}$ & $\begin{array}{c}3 \\
12\end{array}$ & $\begin{array}{c}4 \\
13\end{array}$ & $\begin{array}{c}4 \\
14\end{array}$ & $\begin{array}{c}4 \\
15\end{array}$ \\
\hline Juncus procerus & 60 & 50 & 30 & 60 & 70 & 10 & 60 & 60 & 60 & 80 & 80 & 70 & 60 & 30 & 40 \\
\hline Agrostis capillaris & 15 & 40 & 10 & 30 & 20 & 20 & 50 & 50 & 50 & 20 & 10 & 20 & 30 & 40 & . \\
\hline Mentha pulegium & 20 & + & . & + & + & + & 5 & 5 & 5 & + & . & . & + & + & 60 \\
\hline Lotus uliginosus & 5 & 15 & . & + & + & . & + & 20 & + & . & + & 10 & 20 & 10 & + \\
\hline Leontodon saxatile & . & . & 30 & 10 & 5 & 20 & + & + & + & + & . & . & + & 10 & . \\
\hline Polygonum hydropiperoides & 10 & + & . & . & . & . & . & . & . & . & + & + & + & . & + \\
\hline Holcus lanatus & . & . & + & 10 & 15 & + & . & . & . & + & . & . & . & 10 & . \\
\hline Rumex conglomeratus & + & . & + & . & . & . & + & + & + & + & . & . & . & . & . \\
\hline Juncus imbricatus & . & . & 30 & + & + & 50 & + & + & + & . & . & . & . & . & . \\
\hline Cyperus reflexus & + & . & . & + & . & + & . & . & . & . & . & . & . & . & + \\
\hline Centipeda elatinoides & . & . & . & . & + & . & . & . & . & . & . & + & + & . & . \\
\hline Gratiola peruviana & . & . & . & . & + & . & . & . & . & + & 5 & 5 & . & . & . \\
\hline Prunella vulgaris & . & . & . & . & . & . & + & + & + & + & . & . & . & . & . \\
\hline Eryngium pseudojunceum & + & 5 & . & . & . & . & . & . & . & . & . & . & . & . & + \\
\hline Dichondra sericea & . & . & + & + & + & + & . & . & . & . & . & . & . & . & . \\
\hline Rubus constrictus & . & . & - & + & . & . & + & + & + & . & . & . & . & . & . \\
\hline Polypogon australis & . & . & . & + & + & . & . & . & . & + & . & . & . & . & . \\
\hline Juncus microcephalus & + & . & . & . & . & . & . & . & . & . & + & . & . & . & . \\
\hline Hypochaeris radicata & . & + & + & . & + & . & . & . & . & . & . & . & . & . & . \\
\hline Hydrocotyle marchantioides & . & . & . & . & 5 & + & . & . & . & + & . & . & . & . & . \\
\hline Myrceugenia exsucca & . & . & . & . & . & . & + & $\mathrm{r}$ & $r$ & . & . & . & . & . & . \\
\hline Daucus carota & . & . & + & + & . & . & . & . & . & . & . & . & . & . & . \\
\hline Piptochaetium montevidense & . & . & + & . & . & + & . & . & . & . & . & . & . & . & . \\
\hline Glyceria multiflora & . & . & . & . & . & . & . & . & . & . & + & . & . & . & . \\
\hline Hedyotis salzmannii & . & . & . & . & . & . & . & . & . & . & . & + & . & . & . \\
\hline
\end{tabular}

Además en un censo: Chamomilla recutita, Gamochaeta americana, Plantago lanceolata, Juncus stipulatus, Myriophyllum aquaticum, Ludwigia peploides, Anthoxathum utriculatum, Lotus corniculatus, Phyla nodiflora, Aster vahlii, Cyperus eragrostis, Cuscuta suaveolens, Arhenatherum elatius, Gamochaeta spiciformis, Setaria parvifolia, Baccharis sagittalis, Eleocharis macrostachya, Cyperus conceptionis y Rumex acetosella.

Tabla 2: Estructura fitosociológica del Juncetum procerii. Los valores indican cobertura. Floristic structure of the Juncetum procerii. Data values indicate percentage cover. 
y alta (hasta $120 \mathrm{~cm}$ ) que ocupa el borde de las lagunas. En la tabla formada por 21 censos fitosociológicos se presentan 46 especies, pero el promedio de especies por censo es de 9.57. Esta asociación pratense descrita por Oberdorfer (1960) fue analizada en detalle por San Martín et al. (1991). Montaldo (1975) la considera un climax edáfico antropogénico, mantenida como tal por el pastoreo. Esta asociación vegetal tiene una amplia distribución en toda la depresión intermedia de Chile Central (Valparaíso a Puerto Montt) ocupando depresiones con mal drenaje y la ribera de canales y lagunas.

La especie dominante es el Junquillo (Juncus procerus) que crece acompañado de la Chépica (Agrostis capillaris), el Poleo (Mentha pulegium) y la Alfalfa chilota (Lotus uliginosus). La presencia abundante de Chinilla (Leontodon saxatile) en algunos censos indica degradación del suelo por sobrepastoreo (Ramírez et al., 1991). En lugares muy compactados por el pisoteo del ganado adquiere importancia el Junquillo duro (Juncus

\begin{tabular}{|c|c|c|c|c|c|c|c|c|c|c|c|c|c|c|c|c|}
\hline Laguna: & 1 & 1 & 1 & 1 & 2 & 2 & 4 & 4 & 4 & 5 & 5 & 5 & 6 & 6 & 6 & 7 \\
\hline Especies / Censos: & 1 & 2 & 3 & 4 & 5 & 6 & 7 & 8 & 9 & 10 & 11 & 12 & 13 & 14 & 15 & 16 \\
\hline Mentha pulegium & 60 & 20 & + & 60 & 50 & 70 & 90 & 40 & + & + & 90 & 80 & 60 & 40 & 60 & 30 \\
\hline Polygonum hydropiperoides & 5 & + & + & + & + & 10 & + & + & . & . & + & + & 10 & + & 5 & 10 \\
\hline Leontodon saxatile & + & + & . & 20 & 20 & + & + & + & 70 & 70 & 5 & + & . & . & . & + \\
\hline Agrostis capillaris & 5 & 5 & 5 & 20 & 30 & + & . & . & + & 20 & . & . & & + & . & 15 \\
\hline Lotus uliginosus & 10 & 5 & 80 & + & 5 & . & . & . & + & 10 & + & + & & . & . & 20 \\
\hline Eleocharis pachycarpa & + & 20 & . & + & . & 10 & 5 & . & . & . & 5 & 20 & 30 & 40 & . & . \\
\hline Cyperus reflex us & + & + & . & . & . & + & + & + & . & . & + & + & + & + & . & . \\
\hline Juncus microcephalus & 10 & 10 & + & . & . & . & . & . & . & . & . & + & + & 20 & . & + \\
\hline Rumex conglomeratus & . & . & + & + & + & . & . & . & + & . & + & + & . & . & + & . \\
\hline Phyla nodiflora & . & + & . & . & . & + & . & + & . & . & + & + & + & . & + & . \\
\hline Eleocharis macrostachya & + & . & . & . & . & . & . & . & . & . & . & + & + & + & + & . \\
\hline Gratiola peruviana & . & . & . & . & . & + & . & . & . & . & . & + & + & + & + & . \\
\hline Eryngium pseudojunceum & . & . & 15 & . & & + & . & 5 & . & . & . & + & . & . & . & . \\
\hline Centipeda elatinoides & . & . & . & . & & . & . & . & . & . & + & . & + & + & . & 20 \\
\hline Gnaphalium cy matoides & + & . & . & . & . & . & . & + & . & . & . & . & . & + & 20 & . \\
\hline Rumex acetosella & . & . & . & . & . & . & . & . & + & + & + & . & . & . & . & . \\
\hline Navarretia involucrata & . & . & . & . & . & . & . & . & . & . & . & + & + & + & . & . \\
\hline Eryngium humiffusum & 10 & 40 & . & . & . & . & . & . & . & . & . & . & . & . & . & . \\
\hline Nothoscordum striatellum & + & + & . & . & . & . & . & . & . & . & . & . & . & . & . & . \\
\hline Aster vahlii & & + & . & . & & . & . & . & . & . & . & + & . & . & . & . \\
\hline Polypogon australis & . & . & . & + & + & . & . & . & . & . & . & . & . & . & . & . \\
\hline Glyceria multiflora & . & . & . & . & . & + & + & . & . & . & . & . & . & . & . & . \\
\hline Cirsium vulgare & . & . & . & . & . & . & . & . & 30 & + & . & . & . & . & . & . \\
\hline Cynosurus echinatus & . & . & . & . & . & . & . & . & + & + & . & . & . & . & . & . \\
\hline Gamochaeta americana & . & . & . & . & . & . & . & . & + & + & . & . & . & . & . & . \\
\hline
\end{tabular}

Además en un censo: Juncus imbricatus, Holcus lanatus, Briza minor, Parentucellia viscosa, Cyperus conceptionis, Juncus procerus, Cuscuta suaveolens, Plantago lanceolata, Oxalis corniculata Muehlenbeckia hastulata, Vulpia bromoides, Dichondra sericea, Achillea millefolium, Modiola caroliniana, Prunella vulgaris, Hydrocotyle marchantioides, Sissyrinchium graminifolium, Spergularia rubra, Setaria parvifolia.

Tabla 3. Estructura fitosociológica del Mentho-Agrostietum capilllaris. Los valores indican porcentaje de cobertura. Phytosociological structure of the Mentho-Agrostietum capillaris. Data values indicate percentage cover. 


\begin{tabular}{|c|c|c|c|c|c|c|c|c|c|c|c|}
\hline Laguna: & 2 & 4 & 4 & 4 & 8 & 8 & 9 & 9 & 9 & 10 & 10 \\
\hline Especies / Censos: & 1 & 2 & 3 & 4 & 5 & 6 & 7 & 8 & 9 & 10 & 11 \\
\hline Eleocharis pachycarpa & 60 & 30 & 50 & 90 & 80 & 90 & 95 & 90 & 40 & 40 & 20 \\
\hline Mentha pulegium & + & 70 & 20 & . & . & + & . & + & 10 & + & + \\
\hline Gratiola peruviana & 15 & + & . & . & . & $\mathrm{r}$ & 5 & + & . & 25 & 30 \\
\hline Leontodon saxatile & + & + & + & + & . & . & . & . & + & + & + \\
\hline Polygonum hydropiperoides & 25 & + & . & . & . & + & . & . & 10 & + & . \\
\hline Phyla nodiflora & . & . & + & . & . & + & . & . & + & + & 20 \\
\hline Gnaphalium cymatoides & . & . & + & 5 & . & . & . & . & 20 & 20 & 15 \\
\hline Juncus procerus & . & . & + & 5 & . & + & + & . & . & . & . \\
\hline Cyperus reflexus & . & . & + & . & . & . & . & . & + & + & + \\
\hline Spergularia rubra & . & . & . & . & . & + & . & 5 & + & + & $\cdot$ \\
\hline Glyceria multiflora & . & + & . & . & . & 10 & . & 5 & & . & . \\
\hline Eryngium pseudojunceum & & . & + & . & . & + & + & . & . & . & . \\
\hline Rumex conglomeratus & + & . & . & . & . & + & . & . & . & . & . \\
\hline Myosotis arvensis & . & . & . & + & + & & . & . & . & . & . \\
\hline Lythrum portula & . & . & . & + & . & . & . & . & . & + & . \\
\hline Panicum capillare & . & . & . & + & + & . & . & & . & . & . \\
\hline Polygonum persicaria & . & . & . & $\mathrm{r}$ & + & . & . & . & . & . & . \\
\hline Cyperus eragrostis & . & . & . & . & + & . & . & + & . & . & . \\
\hline
\end{tabular}

Además en un censo: Agrostis capillaris, Lotus uliginosus, Polypogon australis. Centipeda elatinoides, Plantago lanceolata, Juncus microcephalus, Eleocharis macrostachya, Oxalis corniculata, Juncus imbricatus, Alisma plantago-aquatica, Echinochloa crusgalli, Crassula peduncularis, Hypochaeris radicata, Ranunculus bonariensis, Rorippa palustris, Setaria parvifolia.

Tabla 4. Estructura fitosociológica del Eleocharitetum pachycarpae. Los valores indican porcentaje de cobertura. Phytosociological structure of the Eleocharitetum pachycarpae. Data values indicate percentage cover.

imbricatus) y en zonas con mayor anegamiento, el Rime (Eleocharis pachycarpa).

2. Pradera húmeda de Poleo

Mentho-Agrostietum capillaris Contreras, Verdugo, San Martín y Ramírez 1991.

(tab. 3)

Contreras et al. 1991. Flora y vegetación de las praderas húmedas de Chivilcán (Cautín, Chile). Actas II Congreso Internacional Gestión en Recursos Naturales Valdivia 2: 438-455.

La pradera húmeda de Poleo es típica de lugares húmedos, muy pastoreados en la Cordillera de la Costa de la Novena Región de Chile. Se ubica en el borde de las lagunas investigadas, limitando hacia tierra firme con la pradera de Junquillo. Por ello está expuesta a un anegamiento más prolongado. En los meses de verano esta comunidad destaca nítidamente en el paisaje por el color azul de las flores del Poleo (Mentha pulegium) la especie dominante. También son importantes el Duraznillo de agua (Polygonum hydropiperoides) y la Chépica. La presencia abundante del Rime reafirma las mayores condiciones de humedad de los biótopos y la de la Chinilla, que suele hacerse absolutamente dominante (Censos 9 y 10), el sobrepastoreo. La tabla fitosociológica formada por 16 censos contiene un total de 44 especies, pero el promedio de especies por censos alcanza a 9.56.

\section{Pantano de Rime}

Eleocharitetum pachycarpae ass. nov. (Typus inv. $\mathrm{N}^{\circ} 6$ de la tab. 4) 


\begin{tabular}{|c|c|c|c|c|c|c|c|}
\hline Laguna: & 5 & 5 & 5 & 6 & 6 & 6 & 10 \\
\hline Especies / Censos: & 1 & 2 & 3 & 4 & 5 & 6 & 7 \\
\hline Eleocharis macrostachya & 70 & 60 & 80 & 80 & 70 & 55 & 40 \\
\hline Mentha pulegium & 20 & 40 & 20 & + & . & + & . \\
\hline Gratiola peruviana & . & + & & 10 & + & 5 & + \\
\hline Phyla nodiflora & . & + & 5 & & 10 & . & 10 \\
\hline Centipeda elatinoides & . & 10 & 5 & 5 & . & & + \\
\hline Navarretia involucrata & . & . & + & + & . & 30 & \\
\hline Eryngium pseudojunceum & + & . & & + & . & 10 & \\
\hline $\begin{array}{l}\text { Polygonum } \\
\text { hydropiperoides }\end{array}$ & + & . & & . & . & + & \\
\hline Glyceria multiflora & . & + & . & + & . & . & \\
\hline Spergularia rubra & . & . & . & . & . & + & + \\
\hline Glinus radiatus & . & . & . & & + & . & + \\
\hline
\end{tabular}

Además en un censo: Juncus microcephalus, Agrostis capillaris, Lotus ulignosus, Cyperus reflexus, Eryngium humiffusum, Rumex conglomeratus, Eleocharis pachycarpa, Gamochaeta spiciforme, Rumex acetosella, Leontodon saxatile, Gnaphalium cymatoides.

Tabla 5. Estructura fitosociológica del Eleocharitetum macrostachyae. Los valores indican porcentaje de cobertura. Phytosociological structure of the Eleocharitetum macrostachyae. Data values indicate percentage cover.

Esta nueva asociación vegetal pantanosa, es muy abundante en las lagunas estudiadas. Se trata de un pantano bajo $(40 \mathrm{~cm}$ de alto) ampliamente dominado por el Rime, cuya cobertura puede alcanzar hasta el $90 \%$. Generalmente, ocupa la franja que sigue hacia el centro de la laguna, a continuación de la Pradera de Poleo. Coloniza lugares con aguas someras, que en verano se secan completamente, destacándose entonces los tallos postrados del Rime, que tienen un color pardo amarillento. Junto a la especie dominante, crecen los helófitos Duraznillo de agua y la Contrayerba (Gratiola peruviana). También es importante el Poleo.

En los 11 censos levantados en rodales de esta comunidad se presentó un total de 34 especies diferentes, pero el promedio de especies por censo fue de 8.45. Esta comunidad descrita aquí por primera vez, es frecuente en cuerpos acuáticos lénticos del centro-sur de Chile.

\begin{tabular}{|c|c|c|c|c|c|c|c|}
\hline $\begin{array}{l}\text { Laguna: } \\
\text { Especies censos: }\end{array}$ & $\begin{array}{l}4 \\
1\end{array}$ & $\begin{array}{l}4 \\
2\end{array}$ & $\begin{array}{l}4 \\
3\end{array}$ & $\begin{array}{l}4 \\
4\end{array}$ & $\begin{array}{l}6 \\
5\end{array}$ & $\begin{array}{l}7 \\
6\end{array}$ & $\begin{array}{l}7 \\
7\end{array}$ \\
\hline Gnaphalium cymatoides & 15 & 30 & 25 & 40 & 10 & 10 & 60 \\
\hline $\begin{array}{l}\text { Polygonum } \\
\text { hydropiperoides }\end{array}$ & + & 20 & 25 & 30 & 40 & 15 & 10 \\
\hline Cyperus reflexus & + & + & + & + & + & + & \\
\hline Mentha pulegium & + & + & + & 5 & & 10 & 10 \\
\hline Spergularia rubra & & + & 5 & + & 10 & . & + \\
\hline Eleocharis pachycarpa & 20 & + & + & + & . & + & . \\
\hline Centipeda elatinoides & 20 & + & + & + & 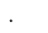 & + & \\
\hline Gratiola peruviana & r. & + & + & + & + & + & \\
\hline Phyla nodiflora & 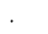 & . & . & + & + & + & + \\
\hline Leontodon saxatile & + & . & . & + & + & . & . \\
\hline Plantago lanceolata & 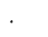 & ${ }^{\circ}$ & + & & + & . & \\
\hline Rumex acetosella & & + & 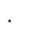 & & . & & + \\
\hline Juncus bufonius & & + & . & + & . & . & \\
\hline Eleocharis macrostachya & & 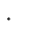 & & & . & + & + \\
\hline
\end{tabular}

Además en un censo: Oxalis corniculata, Chamomilla recutita, Polygonum persicaria, Navarretia involucrata, Glynus radiatus.

Tabla 6. Estructura fitosociológica del GnaphalioPolygonetum. Los valores indican porcentaje de cobertura. Phytosociological structure of the Gnaphalio-Polygonetum. Data values indicate percentage cover.

\section{Pantano de Rime Grande}

Eleocharitetum macrostachyae Contreras, Verdugo, San Martín y Ramírez 1991.

(tab. 5)

Contreras et al. 1991. Flora y vegetación de las praderas húmedas de Chivilcán (Cautín, Chile). Actas II Congreso Internacional Gestión en Recursos Naturales Valdivia 2: 438-455.

Esta asociación vegetal se presentó en tres lagunas de las estudiadas. En ella domina el Rime grande (Eleocharis macrostachya) acompañado del Poleo, con menor importancia. La presencia en él de Hierba de la virgen, indica intervención con pastoreo de ganado doméstico. Cuando hay condiciones de anegamiento prolongado aparece la Contrayerba. De las 22 especies presentes en los 7 censos de vegetación levantados, sólo se presenta un promedio de 7.15 especies por censo. En la zonación litoral de las lagunas estudiadas, esta asociación vegetal marca el límite entre la orilla y el centro de la laguna. Se 


\begin{tabular}{llllllll}
\hline Laguna: & 3 & 3 & 3 & 3 & 3 & 3 & 3 \\
Especies / Censos: & 1 & 2 & 3 & 4 & 5 & 6 & 7 \\
\hline Sagittaria montevidense & 20 & 50 & 70 & 80 & 40 & 80 & 70 \\
Ludwigia peploides & 10 & + & + & + & + & 10 & 5 \\
Callitriche terrestris & + & + & + & + & + & + &. \\
Limosella australis & + &. & + &. & + & + & + \\
Scirpus californicus & 30 & 20 & 20 & + &. &. &. \\
Utricularia gibba & + & + &. &. &. & + & + \\
Callitriche autumnalis &. &. &. &. &. & + & +
\end{tabular}

Ademas en sun censo: Lythrum portula, Myriophyllum aquaticum, Gratiola peruviana.

Tabla 7. Estructura fitosociológica de la comunidad de Ludwigia peploides-Sagittaria montevidense. Los valores indican porcentaje de cobertura. Phytosociological structure of the Ludwigia peploides-Sagittaria montevidense comunity. Data values indicate percentage cover.

presenta en forma abundante en lagunas y canales de regadío en la provincia de Cautín.

\section{Prados de Vira-Vira celeste}

Gnaphalio-Polygonetum hydropiperoidis ass. nov.

\section{(Typus Censo $\mathrm{N}^{\circ} 4$ de la tabla 6)}

Esta comunidad pratense es exclusiva de las lagunas primaverales estudiadas. Ella se presenta en el centro de las lagunas, ocupando lugares denudados estacionalmente, con prolongada sequía estival y cubiertos con sedimento fino. Se trata de una comunidad vegetal muy homogénea, abierta, con una cobertura total que no supera el $70 \%$ y en la cual abundan hierbas pioneras anuales. La especie dominante es la hierba nativa ViraVira celeste (Gnaphalium cymatoides) una Asteraceae de un color verde claro, casi celeste, que destaca desde lejos entre la vegetación circundante. Le acompañan el Duraznillo de agua, la Cortadera chica (Cyperus reflexus) y el Poleo. La Peorrilla (Centipeda elatinoides) y la Contrayerba indican sin embargo, la presencia de humedad durante gran parte del año. De las 19 especies que forman la tabla fitosociológica, se presenta sólo un promedio de 9.42 especies por censo.

\begin{tabular}{llllll}
\hline Laguna: & 8 & 8 & 8 & 8 & 8 \\
Especies / Censos: & 1 & 2 & 3 & 4 & 5 \\
\hline Lythrum portula & 40 & 80 & 80 & 70 & 90 \\
Eleocharis pachycarpa & 40 & 15 & 10 & 20 & + \\
Glynus radiatus &. & + & + & + & + \\
Gnaphalium cymatoides & 20 & + & 5 &. &. \\
Alisma plantago-aquatica &. &. & + & + & + \\
Crassula peduncularis &. & + & + & + &. \\
Centipeda elatinoides & + &. &. & 5 &. \\
Veronica anagallis-aquatica & + &. &. &. & + \\
Callitriche terrestris &. & + &. &. & 10 \\
Juncus dombeyanus & $\cdot$ &. & + & 5 &.
\end{tabular}

Además en un censo: Juncus procerus, Mentha pulegium, Polygonum hydropiperoides, Myosotis arvensis, Polygonum persicaria, Cyperus eragrostis.

Tabla 8. Estructura fitosociológica de la comunidad de Eleocharis pachycarpa-Lythrum portula. Los valores indican porcentaje de cobertura.Phytosociological structure of the Eleocharis pachycarpa-Lythrum portula comunity. Data values indicate percentage cover.

\section{Pantano de Rosa de agua \\ Comunidad de Ludwigia peploides-Sagittaria montevidense}

(tab. 7)

Esta comunidad vegetal se presenta cubriendo toda la parte central de la laguna $\mathrm{N}^{\circ}$ 3. Se trata de una comunidad pantanosa de aguas someras, que a fines del verano de 1997 se secó completamente. La especie dominante es el helófito Rosa de agua (Sagittaria montevidense), acompañada de la hierba natante Clavito de agua (Ludwigia peploides) y de la especie sumergida Bolsita de agua (Utricularia gibba). En algunos censos se presentó la Totora (Scirpus californicus) en forma abundante, evidenciando un avance del hidrosere (Steubing et al., 1980). El total de especies presentes en la Tabla fitosociológica fue de 10 y el promedio, de 5.42 especies por censo.

7. Prados de Romerillo rojo y Rime Comunidad de Eleocharis pachycarpaLythrum portula

(tab. 8) 


\begin{tabular}{lcccc}
\hline Laguna: & 6 & 7 & 10 & 10 \\
Especies / Censos: & 1 & 2 & 3 & 4 \\
\hline Anthoxanthum utriculatum & 70 & 40 & 40 & 70 \\
Juncus procerus & 10 & 20 & 20 & 10 \\
Leontodon saxatile & 10 & 30 & 30 & 10 \\
Mentha pulegium & + & + & + & + \\
Holcus lanatus & + & + & + & + \\
Hypochaeris radicata & + & + & + & + \\
Prunella vulgaris & + & + & + & + \\
Rumex acetosella & + & + & + & + \\
Rubus constrictus & + & + & + & + \\
Crepis capillaris & + & $\cdot$ & $\cdot$ & + \\
Dichondra sericea & + & $\cdot$ & $\cdot$ & + \\
Juncus imbricatus & + & $\cdot$ & $\cdot$ & + \\
Lotus uliginosus & & + & + & \\
Hedyotis salzmannii &. & + & + &. \\
Geranium core-core & $\cdot$ & + & + & $\cdot$ \\
Gamochaeta americana & $\cdot$ & + & + & $\cdot$ \\
Trifolium repens & $\cdot$ & + & + & $\cdot$ \\
Paspalum distichum & $\cdot$ & $\mathrm{r}$ & + &. \\
\hline
\end{tabular}

Además en un censo: Agrostis capillaris, Hydrocotyle marchantioides.

Tabla 9. Estructura fitosociológica de la comunidad de Anthoxanthum utriculatum. Los valores indican porcentaje de cobertura. Phytosociological structure of the Anthoxanthum utriculatum comunity. Data values indicate percentage cover.

Esta asociación vegetal sólo se presentó en la laguna $\mathrm{N}^{\circ} 8$, que fue secada para facilitar una construcción. Se presenta en la parte más somera del centro de la laguna y se desarrolla cuando termina el anegamiento. Se trata de una comunidad vegetal muy densa, con una alta cobertura. La especie dominante fue el Romerillo rojo (Lythrum portula) acompañada del Rime. Algunos censos practicados en sectores con mayor sequía, comenzaban a ser invadidos por la Vira-Vira celeste. El promedio fue de 7.4 especies por censo y el total de la tabla de 16 especies. Una asociación similar ha sido descrita recientemente en un basural de Osorno (Chile) por Ramírez et al. (1996).

8. Pradera alta de Paja ratonera Comunidad de Anthoxanthum utriculatum (tab. 9)

Esta asociación vegetal pratense está ampliamente distribuida en las Novena y

\begin{tabular}{lllll}
\hline Laguna: & 5 & 10 & 10 & 10 \\
Especies / Censos: & 1 & 2 & 3 & 4 \\
\hline Phyla nodiflora & 90 & 99 & 90 & 99 \\
Eleocharis macrostachya & + & $\cdot$ & 10 & + \\
Setaria parvifolia & $\cdot$ & + & + & + \\
Rumex acetosella &. & + &. & + \\
Leontodon saxatile & + & + &. &. \\
\hline
\end{tabular}

Además en un censo: Rumex conglomeratus, Mentha pulegium, Cyperus reflexus, Chamomilla recutita, Polygonum aviculare.

Tabla 10. Estructura fitosociológica de la comunidad de Phyla nodiflora. Los valores indican porcentaje de cobertura.Phytosociological structure of the Phyla nodiflora comunity. Data values indicate percentage cover.

Decima Regiones de Chile. Una asociación similar había sido descrita por San Martín et al. (1992) como una subasociación halofítica del Centello-Agrostietum capillaris, para las marismas del centro-sur de Chile. La tabla confeccionada en este trabajo, entrega una composición florística diferente, que ha sido denominada Comunidad de Anthoxanthum utriculatum y que correspondería a una comunidad de condiciones más glicófilas.

La especie dominante es la Paja ratonera (Anthoxanthum utriculatum) que crece acompañada de Junquillo y Chinilla, que la señalan como una comunidad degradada originada a partir del Juncetum procerii, por el sobrepastoreo con ovejas o por cultivos reiterados. Su condición de secundaria la confirman la presencia de la Hierba del chancho (Hypochaeris radicata) y de la Zarzamora (Rubus constrictus). A pesar de ser una asociación de lugares degradados, la tabla fitosociológica muestra una gran homogeneidad y un alto promedio de especies por censo, que alcanza a 14. Esta tabla formada por sólo 4 censos, presenta un total de 20 especies, la mayoría malezas de origen alóctono.

\section{Prados de Hierba de la virgen} Comunidad de Phyla nodiflora

(tab. 10) 
Esta comunidad vegetal pratense fue detectada en las lagunas números 5 y 10 , ocupando el centro compactado de ellas, por el pisoteo del ganado. Es una comunidad muy pobre en especies, que sin embargo, cubre totalmente el suelo. Todas las especies que la forman muestran escaso desarrollo. La especie dominante es la Hierba de la virgen, que siempre cubre más del $90 \%$ y que mostrando un crecimiento arrosetado permanece adherida al suelo. La presencia de Eleocharis macrostachya señala condiciones de humedad. En los 4 censos levantados se encontró un total de 10 especies, con un promedio de 4,75 especies por censo.

\section{Comunidad natante de Gliceria}

Glycerietum multiflorae Contreras, Verdugo, San martín, Ramírez 1991

Contreras et al. 1991. Flora y vegetación de las praderas húmedas de Chivilcán (Cautín, Chile). Actas II Congreso Internacional Gestión en Recursos Naturales Valdivia 2: 438-455.

Los dos censos levantados en la laguna número 3 (Glyceria multiflora 30, 20; Sagittaria monvidense 20, 20; Ludwigia peploides 10, 10; Agrostis Capillaris +, .; Lythrum portula ., +; Callitriche verna ., +) concuerdan con la asociación descrita por Contreras et al. (1991) para las Vegas de

\begin{tabular}{ccccc}
\hline Laguna & Superficie (ha) & Censos & Especies & Comunidades \\
\hline 1 & 0.27 & 7 & 19 & 3 \\
2 & 0.45 & 8 & 27 & 3 \\
3 & 3.60 & 11 & 21 & 3 \\
4 & 2.88 & 14 & 36 & 4 \\
5 & 0.10 & 6 & 25 & 3 \\
6 & 0.90 & 10 & 33 & 5 \\
7 & 0.15 & 6 & 34 & 4 \\
8 & 0.32 & 10 & 31 & 4 \\
9 & 0.36 & 3 & 13 & 1 \\
10 & 0.90 & 11 & 35 & 5 \\
\hline
\end{tabular}

Tabla 11. Tamaño de las lagunas investigadas, número de censos levantados en cada una y especies y comunidades vegetales encontradas en ellas. Size, number of inventories, species and communities found in the studied lagoons.
Chivilcán. Se trata de una comunidad acuática estacionalmente natante, formada por varias especies palustres. Domina el pasto Glyceria multiflora, acompañado de la Rosa de agua y el Clavito de agua. Esta comunidad tiene pocas especies, ya que en los dos censos prospectados, sólo se encontraron 6 especies con un promedio de 4,5 especies por censo.

\section{Pantano de Cortadera azul y Junquillo}

Junco-Caricetum ripariae San Martín 1992

San Martín, C. (1992). Flora, vegetación y dinámica vegetacional de la laguna Santo Domingo (Valdivia, Chile). Tesis, Escuela de Graduados, Universidad Austral de Chile, Valdivia. 192 pp.)

Este pantano es muy frecuente en la región de Valdivia, más al Sur del lugar de estudio. En las lagunas investigadas, por el contrario, es muy escasa y sólo se presentó en un sólo censo (Carex riparia 90; Agrostis capillaris 10; Juncus procerus 5; Mentha pulegium +; Polygonum hydropiperoides +; Rumex conglomeratus +) de la laguna número 1. En este pantano dominan la Cortadera azul (Carex riparia) y la Chépica. También es importante el Junquillo. Se trata de una comunidad pantanosa de alta humedad edáfica y pocas especies. No es posible entregar más detalles, debido a que sólo se levantó un censo en ellas (Ver tabla 1).

\section{Comunidad sumergida de Canehuín Comunidad de Potamogeton pusillus}

Esta asociación vegetal monoespecífica, dominada por Potamogeton pusillus no ha sido aún descrita en Chile, a pesar de ser bastante

\begin{tabular}{lccc}
\hline & Censos & Especies & Comunidades \\
\hline Superficie & 0.69 & 0.09 & -0.84 \\
Censos & & 0.65 & 0.67 \\
Especies & & & 0.89
\end{tabular}

Tabla 12. Análisis de regresión líneal. Valores de r. Lineal regression analysis. Values of $r$. 
Asociación o Comunidad/ Lagunas:

$\begin{array}{lllllllllll}1 & 2 & 3 & 4 & 5 & 6 & 7 & 8 & 9 & 10 & \mathrm{~F}\end{array}$

\section{Juncetum procerii}

Mentho-Agrostietum capillaris

Eleocharitetum pachycarpae

Gnaphalio-Polygonetum hydropiperoidis

Eleocharitetum macrostachyae

de Anthoxanthum utriculatum

de Phyla nodiflora

Junco-Caricetum ripariae

Glycerietum multiflorae

de L. peploides-S. montevidense

de E. pachycarpa-L. portula

de Potamogeton pusillus

Total asociaciones o comunidades

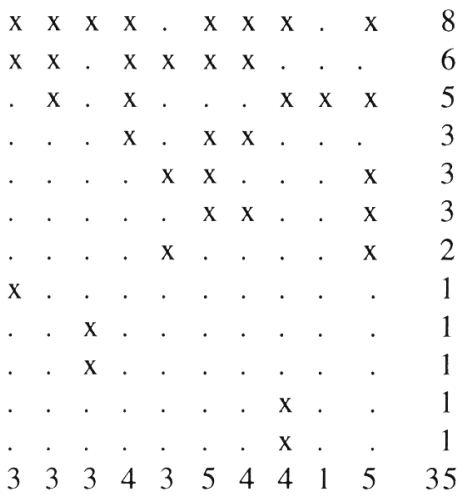

Tabla 13. Presencia de las asociaciones en las lagunas investigadas. Ab. = Abreviatura de las asociaciones. $\mathrm{F}=$ frecuencia. Presence of associations in the studied lagoons. Ab=Associations abbreviations. $F=$ Frecuency.

frecuente en lagunas interiores, de la depresión intermedia de todo Chile Central. Posiblemente sea un homónimo de un Potametum pusillae europeo, lo que deberá ser confirmado a futuro. No es posible entregar mayores detalles por haber prospectado sólo un rodal, en la laguna número 8 (tab. 1). En el único censo levantado sólo aparecía Potamogeton pusillus con $100 \%$ de cobertura.

\section{Número de especies}

La tabla 11 muestra la superficie, el número de censos levantados y el número de especies vegetales presentes en cada laguna. El análisis de regresión lineal aplicado a ella demostró una relación positiva entre la superficie y el número de censos y, entre éstos, y el número de especies y asociaciones vegetales. Entre la superficie de la laguna y el número de asociaciones se presentó una relación negativa; mientras que entre el número de especies y el número de asociaciones, se encontró una alta relación positiva (tab. 12). Lo anterior indica que las diferencias entre las lagunas se deben más a las condiciones del hábitat, que al tamaño de las mismas.

\section{Distribución de asociaciones y comunidades}

Ninguna laguna presentó las 12 asociaciones vegetales descritas. Las lagunas números 6 y 10 presentaron un máximo de 5 asociaciones cada una y la laguna número 9 , presentó sólo una. Además hay tres lagunas con 4 asociaciones y cuatro, con 3 (tab. 13).

De las 12 asociaciones descritas la más frecuente fue el Juncetum procerii que se presentó en 8 de las 10 lagunas investigadas. El Mentho-Agrostietum capillaris se presentó en 6 lagunas y el Eleocharitetum pachycarpae en 5. El Gnapahalio-Polygonetum hydropiperoidis, el Eleocharitetum macrostachyae y la comunidad de Anthoxanthum utriculatum se presentaron en tres lagunas. Por último, la comunidad de Phyla nodiflora se encontró en dos lagunas. Además, hay 5 asociaciones o comunidades que sólo se encontraron en una laguna.

El dendrograma de la figura 3 que muestra 


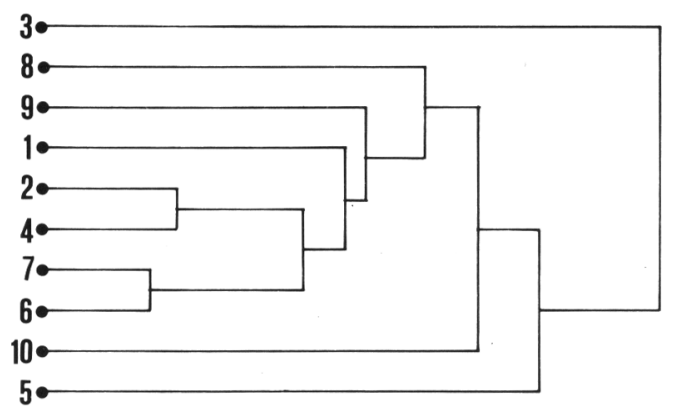

Figura 3. Dendrograma de similitud vegetacional entre las lagunas. Dendrogram of vegetational similitude among the lagoons.

la afinidad fitosociológica entre las lagunas, indica una gran heterogeneidad entre ellas. Así, con un $80 \%$ de afinidad se obtienen dos conglomerados de dos lagunas cada uno (2 y 4, 6 y 7) y 6 lagunas aisladas, con escasa afinidad fitosociológica entre ellas y con los conglomerados descritos. La heterogeneidad vegetacional descrita depende en primer lugar de la duración de la sequía estival que presenta cada laguna.

\section{Origen fitogeográfico}

Al considerar el número de especies, en 7 asociaciones o comunidades vegetales (Juncetum procerii, Mentho-Agrostietum capillaris, Eleocharitetum macrostachyae, Ludwigia peploides-Sagittaria montevidense, Eleocharis pachycarpae-Lythrum portula, Glycerietum multiflorae y de Potamogeton pusillus) dominan las especies nativas y en 5 (Eleocharitetum pachycarpae, GnaphalioPolygonetum hydropiperoides, de Anthoxanthum utriculatum, de Phyla nodiflora y Junco-Caricetum ripariae), las introducidas (fig. 4).

Al considerar la cobertura de los individuos de cada especie, en 10 asociaciones dominan las especies nativas y sólo en el Mentho-Agrostietum capillaris y la comunidad de Eleocharis pachycarpa-Lythrum portula, las alóctonas. De manera que en las vegetación de las lagunas primaverales estudiadas dominan las especies autóctonas, lo que le otorga un carácter de primario (original), con excepción del Mentho-Agrostietum capillaris y de la comunidad de Eleocharis pachycarpa-Lythrum portula, que serían secundarias. La comunidad de Anthoxanthum utriculatum, reconocida como secundaria, presentó una dominancia de especies alóctonas, que sin embargo, es superada por la cobertura de las especies nativas dominantes.

\section{Espectro biológico}

Sólo los espectros biológicos del Juncetum procerii y del Mentho-Agrostietum capillaris son completos, ya que incluyen todas las formas de vida. La presencia de fanerófitos en ellos se debe a su estrecha relación con el bosque pantanoso original de Temo-Pitra (Blepharocalyo-Myrceugenietum exsuccae), al que reemplazan después de su tala (fig. 5).

Un espectro biológico con 4 formas de vida presentan el Eleocharitetum pachycarpae y las comunidades de Eleocharis pachycarpaeLythrum portula y de Anthoxanthum utriculatum. Esta última comunidad, incluye fanerófitos, mostrando también cierta relación con el bosque original y con las praderas de Junquillo y de Poleo. Con tres formas de vida se presentan el Eleocharitetum macrostachyae, el Gnaphalio-Polygonetum y la comunidad de Phyla nodiflora, las tres con un espectro biológico muy parecido, donde dominan hemicriptófitos, caméfitos y terófitos, indicando condiciones menos favorables, seguramente por un período de sequía más prolongado. Con dos formas de vida aparecen la comunidad de Ludwigia peploides-Sagittaria montevidense y las asociaciones JuncoCiperetum ripariae y Glycerietum multiflorae. En los dos primeros dominaron criptófitos palustres y en el último, los hemicriptófitos. 


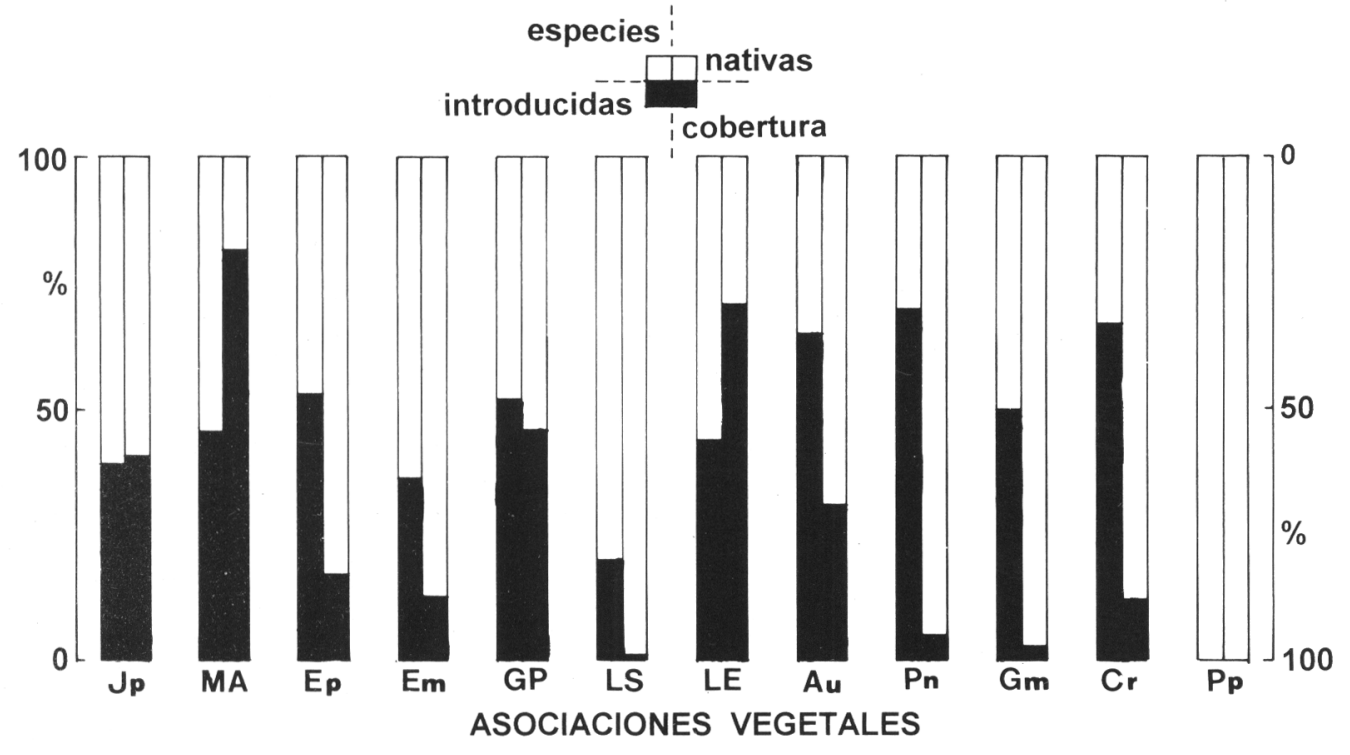

Figura 4. Espectro de origen de la flora de las asociaciones descritas. Abreviaturas de las asociaciones en la tabla 1. Origin spectrum of the flora of the described plant associations. Abbreviations as in table 1.

Por último, en la comunidad de Potamogeton pusillus sólo hay una forma de vida: criptófitos sumergidos. La reducción de los espectros biológicos indica condiciones extremas de biótopos poco favorables para el desarrollo vegetal (Hauenstein et al., 1988).

En la mayoría de los espectros dominan hemicriptófitos, indicando condiciones de intervención humana. La presencia de caméfitos señala condiciones desfavorables y la de los terófitos, un prolongado período de sequía estival (Ramírez et al., 1994).

Al considerar la cobertura todos los espectros biológicos se simplifican, reduciendo considerablemente sus formas de vida, ya que algunas de ellas no alcanza al $1 \%$ de representatividad (fig. 6). En este caso, sólo la comunidad de Anthoxanthum utriculatum presentó 4 formas de vida, el resto tres y menos. Ahora se hace muy nítida la importancia de los terófitos (plantas anuales) en el GnaphalioPolygonetum y en la comunidad de Eleocharis
pachycarpa-Lythrum portula, que ocupan el centro seco de las lagunas, durante primavera y verano. En la mayoría de los espectros biológicos aumentó la cobertura de los hemicriptófitos. Los caméfitos redujeron su importancia con excepción del MenthoAgrostietum capillaris, en el cual aumentaron. Las asociaciones hidrófilas palustres, natantes y sumergidas cambiaron muy poco su espectro al considerar la cobertura, debido a la escasa cantidad de especies que conforman su flora (San Martín et al., 1993).

\section{Análisis estadístico multivariante}

La mayor similitud florística se presentó entre el Glycerietum multiflorae y la comunidad de Ludwigia peploides-Sagittaria montevidense, que forman el conglomerado más consistente (fig. 7). Un segundo grupo lo constituyen la comunidad de Eleocharis pachycarpa-Lythrum portula y el 

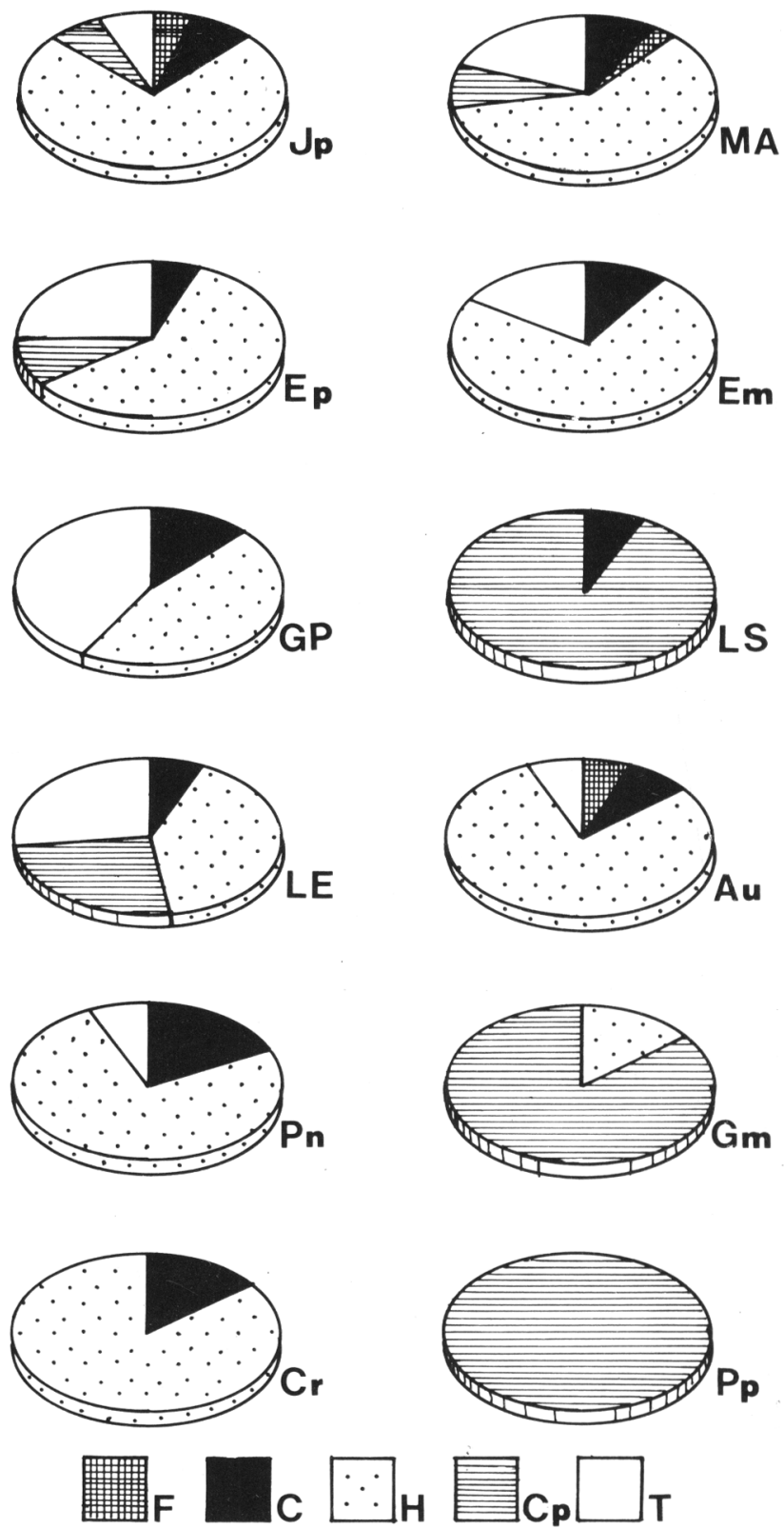

FORMAS DE VIDA

Figura 5. Espectro biológico de la flora de las asociaciones descritas, considerando el número de especies por forma de vida. Abreviaturas de las asociaciones en tabla 1. Formas de vida: $\mathrm{F}=$ fanerófitos, $\mathrm{C}=$ caméfitos, $\mathrm{H}=$ hemicriptófitos, $\mathrm{Cp}=$ criptófitos y $\mathrm{T}$ = terófitos. Biological spectrum of the flora of the described plant associations, considering the species number for each life form. Abbreviations of the associations as in table 1 . Life forms: $F=$ phanerophytes, $C=$ chamaephytes, $H=$ hemicryptophytes, $C p=$ cryptophytes an $T=$ therophytes. 

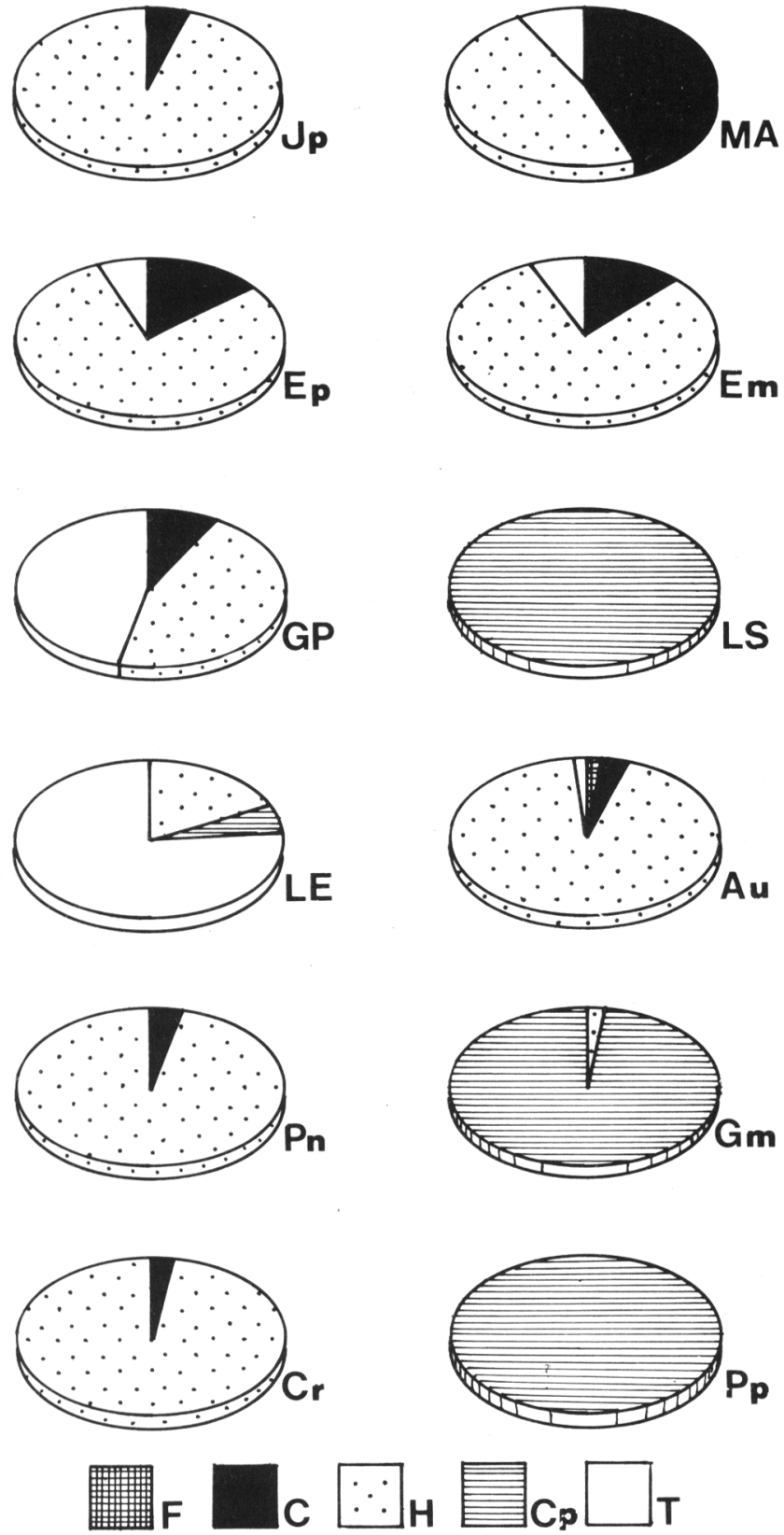

FORMAS DE VIDA

Figura 6. Espectro biológico de la flora de las asociaciones descritas, considerando la cobertura total de cada forma de vida. Abreviaturas de las asociaciones en tabla 1 y de las formas de vida, en figura 5. Biological spectrum of the flora of the described associations, considering the cover for each life forms. Abbreviations of the associations as in table 1 and of the life forms as in figure 5. 


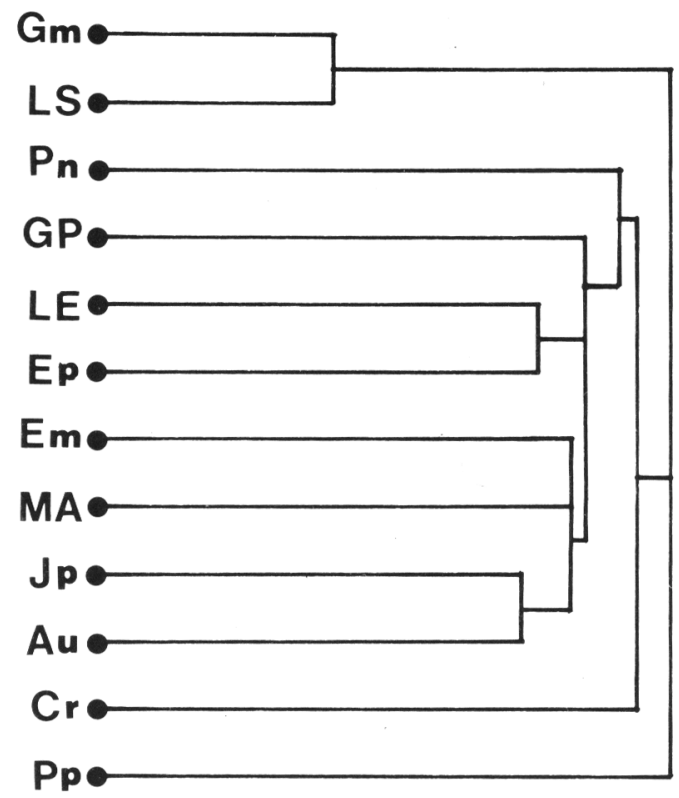

Figura 7. Dendrograma de similitud florística entre las asociaciones descritas. Abreviaturas en tabla 1. Dendogram of floristic similarity among the described plant associations. Abbreviations as in table 1 .

Eleocharitetum pachycarpae, que comparten el Rime. Un tercer conglomerado lo forman el Juncetum procerii y la comunidad de Anthoxanthum utriculatum, confirmando la afinidad florística y ecológica entre ellos. Se ha postulado que esta última asociación reemplazaría a la primera por degradación del suelo (San Martín et al., 1992). Este conglomerado se une con igual similitud al Mentho-Agrostietum capillaris y al Eleocharitetum macrostachyae, señalando una relación dinámica entre dichas asociaciones vegetales y también su vecindad en la zonación litoral. El resto de las asociaciones, aparecen aisladas en el dendrograma.

Al ordenar las especies en los tres primeros componentes principales, que explican el $35.87 \%$ de la variación total, se produce una concentración de ellas en el extremo derecho de la Figura 8. De las especies segregadas fuera de esa zona de concentración, Mentha pulegium, Sagittaria mondevidense y Potamogeton pusillus, se ubican en los extremos alejándose mucho entre sí. La primera estaría indicando condiciones propias de praderas húmedas, la segunda anegamiento estacional y la tercera, anegamiento permanente. En este arreglo la posición de Gnaphalium cymatoides, que crece en la época estival en el centro seco de las lagunas, representa condiciones típicas de praderas secas. El tercer componente, ubica con poca altura en el diagrama a Agrostis capillaris, Leontodon saxatile y sobre todo a Anthoxanthum utriculatum, todas indicadoras de degradación del suelo (Ramírez et al., 1991). Seguramente este componente representa un gradiente de materia orgánica en el sustrato, que aumentaría hacia arriba.

Al ordenar las asociaciones en los tres primeros componentes principales se obtiene el gráfico de la Figura 9. En él se observa que, el Glycerietum multiflorae y la comunidad de Ludwigia peploides-Sagittaria montevidense, comunidades acuáticas del tipo natante, se ubican en el extremo izquierdo, donde según el ordenamiento específico presentado anteriormente, existirían condiciones de anegamiento estacional. El resto de las asociaciones se ordenan en el extremo derecho del diagrama disponiéndose en un claro gradiente de anegamiento que aumenta desde adelante hacia atrás. Este gradiente, es fácilmente asimilable a la zonación litoral de las lagunas estudiadas.

\section{Zonación y Dinámica vegetacional}

Las asociaciones vegetales descritas se disponen en una zonación litoral en la cual cada una ocupa una posición característica, de acuerdo a sus requerimientos de humedad. Un esquema de una laguna ideal con la zonación completa, incluyendo su alteración por la acción antrópica, se presenta en la figura 10. 


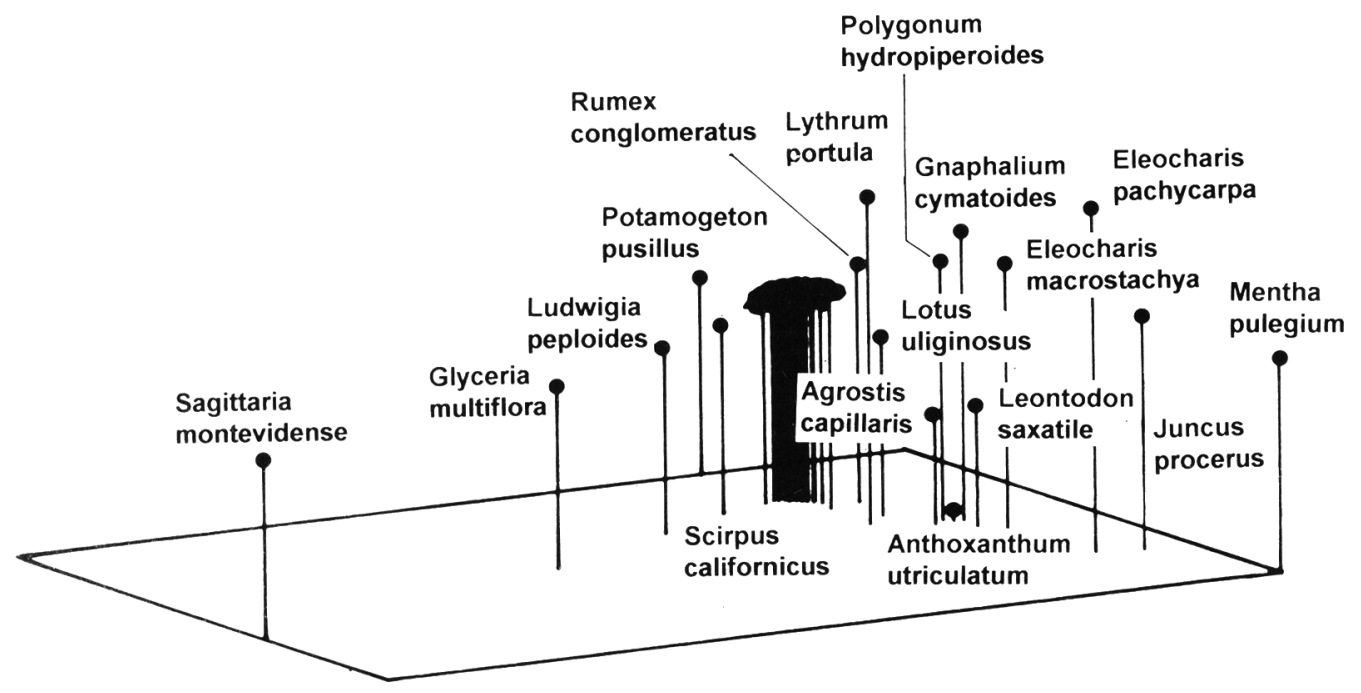

Figura 8. Distribución espacial de las especies en los tres primeros componentes principales. Spatial distribution of the species in the first three principal components.

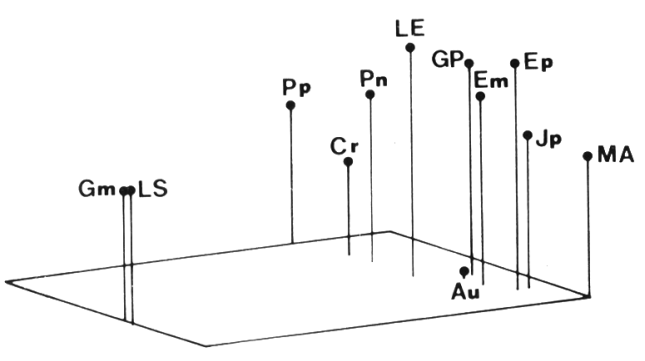

Figura 9. Distribución espacial de las asociaciones descritas en los tres primeros componentes principales. Abreviaturas en tabla 1. Spatial distribution of the described associations in the first three principal components. Abbreviations as in table 1.

El límite externo y más alto en la gradiente litoral está ocupado por el Juncetum procerii (Pradera húmeda de Junquillo). En lugares donde este cinturón vegetacional ha sido cultivado en forma reiterada, es reemplazado por la Pradera alta de Paja ratonera (Anthoxanthum utriculatum). Hacia el interior de la laguna continúa la Pradera de Poleo (Mentho-Agrostietum capillaris) que indica un pastoreo prolongado. En litorales más húmedos y con menor intervención se presenta aquí un Pantano de Cortadera azul (Junco-Caricetum ripariae). Más hacia el centro de la laguna se ubica el Pantano de Rime (Eleocharitetum pachycarpae) que en condiciones de anegamiento más prolongado, suele convivir con la vegetación natante del Glycerietum multiflorae. Por último, el límite inferior de la zonación litoral es ocupado por el Pantano de Rime grande (Eleocharitetum macrostachyae).

El centro de las lagunas puede ser ocupado por varias asociaciones vegetales que se reemplazan mutuamente según la duración de la sequía estival y la intensidad del pastoreo. Así en condiciones de anegamiento permanente aparece la comunidad sumergida de Huiro (Potamogeton pusillus). En lagunas más someras, en este lugar se sitúa el Pantano de Rosa de agua (Ludwigia peploides-Sagittaria montevidense). En lagunas con sequía estival aparecen Prados de Romerillo rojo (Eleocharis 


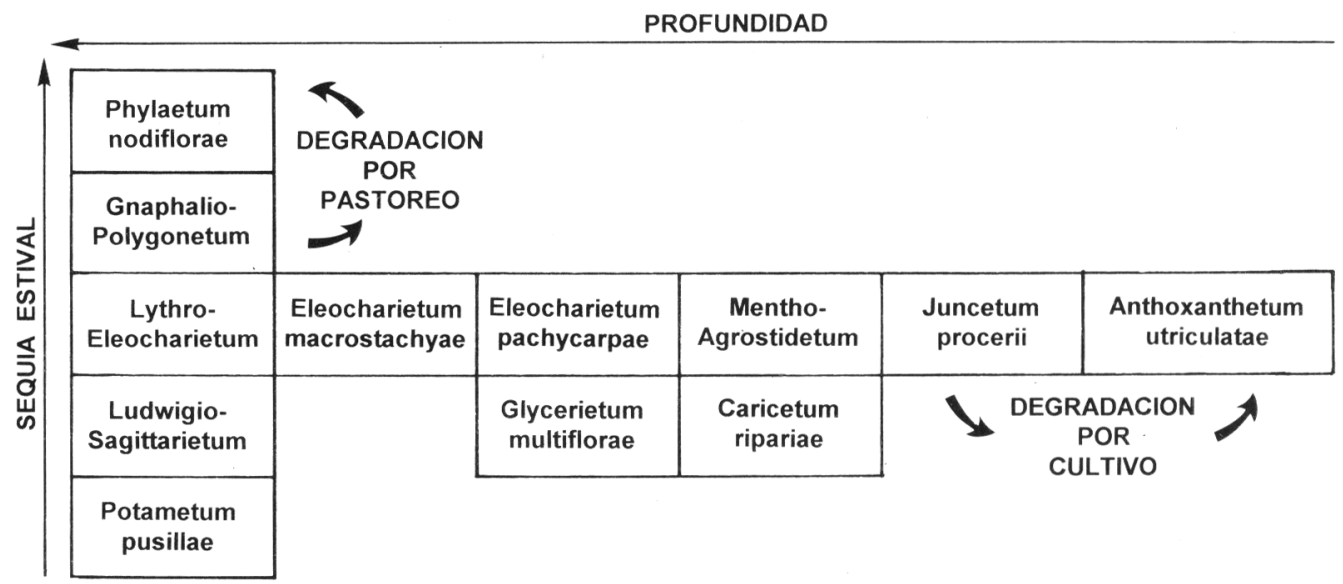

Figura 10. Disposición de las asociaciones descritas en la zonación del gradiente litoral en una laguna en que convivieran todas. Location of the described plant associations in the gradient litoral zonation in an ideal lagoon with all the associations.

pachycarpa-Lythrum portula) que en condiciones de mayor sequía son reemplazados por un Prado de Vira-Vira celeste (GnaphalioPolygonetum). Por último, esta comunidad a su vez puede ser reemplazada por un prado de Hierba de la virgen (Phyla nodiflora) ante una mayor eutroficación y sequía edáfica provocada por compactación.

\section{CONCLUSIONES}

De los resultados descritos y discutidos en los capítulos anteriores, se pueden extraer las siguientes conclusiones:

1) Existe escasa similitud vegetacional entre las lagunas primaverales investigadas debido más a la heterogeneidad ambiental, antes que al tamaño de las mismas.

2) La alta heterogeneidad ambiental permite la existencia de 12 asociaciones vegetales correspondientes a formaciones pratenses, pantanosas y acuáticas.

3) De las 12 asociaciones determinadas
Juncetum procerii, Mentho-Agrostietum capillaris, Glycerietum multiflorae, JuncoCaricetum ripariae y Eleocharitetum macrostachyae habían sido descritas anteriormente. 4) Eleocharitetum pachycarpae, GnaphalioPolygonetum hydropiperoidis, Ludwigia peploides-Sagittaria montevidense, Eleocharis pachycarpa-Lythrum portula y las comunidades de Anthoxanthum utriculatum, de Phyla nodiflora y de Potamogeton pusillus son asociaciones o comunidades nuevas para la ciencia que no habían sido aún descritas.

5) En la mayoría de las asociaciones domina el elemento florístico autóctono y sólo en el Mentho-A grostietum capillaris y la comunidad de Eleocharis pachycarpa- Lythrum portula, el alóctono.

6) Se constató una gran simplificación del espectro biológico en las diferentes comunidades vegetales, impuesta tanto por la heterogeneidad ambiental, como también por la pobreza en especies de las asociaciones.

7) En la zonación litoral se determinaron 7 asociaciones pratenses, pantanosas y acuática natantes y, aunque ellas no están presentes en 
todas las lagunas, siempre aparecen en la misma secuencia y posición espacial en el gradiente litoral.

8) Cinco asociaciones integran la vegetación del centro de las lagunas, las cuales se reemplazan según la duración del período de sequía estival y la intensidad de la intervención antrópica.

9) La afinidad florística entre las diferentes asociaciones vegetales descritas, refleja su proximidad espacial en la zonación litoral.

10) La distribución de las asociaciones en las diferentes lagunas está condicionada por la duración del anegamiento, la profundidad del agua, el contenido en materia orgánica del sustrato y por la degradación del mismo provocada por el pastoreo.

\section{BIBLIOGRAFÍA}

BARKMANN, J., C. MORAVEC \& S. RAUSCHERT -1986- Code of phytosociological nomenclature. Vegetatio 67: 145-195.

BESOAIN, M. - 1985- Los suelos. En: J. Tosso (Ed.) Suelos volcánicos de Chile. INIA, Ministerio de Agricultura. Santiago.

CONTRERAS, D., M. VERDUGO, J. SAN MARTIN y C. RAMÍREZ - 1991- Flora y vegetación de las praderas húmedas de Chivilcán (Cautín, Chile). Actas II Congreso Internacional Gestión en Recursos Naturales Valdivia 2: 438-455.

DI CASTRI, F. y E. HAJEK -1976- Bioclimatología de Chile. Universidad Católica de Chile. Santiago.

DIERSCHCKE, H. -1994- Pflanzensoziologie: Grundlagen und Methoden. E. Ulmer. Stuttgart.

DIGBY, P.G. \& R.A. KEMPTON -1987Multivariate analysis of ecological communities. Chapman \& Hall. New York.

ELLENBERG, H. -1956- Aufgaben und Methoden der Vegetationskunde. Einführung in die Phytologie 4: 1-136.

GAUCH, H. -1982- Multivariate analysis in community ecology. Cambridge University Press. Cambridge.

HAJEK, E. y F. DI CASTRI -1975-Bioclimatografía de Chile. Universidad Católica de Chile.
Santiago.

HAUENSTEIN, E., C. RAMIREZ, M. LATSAGUE y D. CONTRERAS -1988- Origen fitogeográfico y espectro biológico como medida del grado de intervención antrópica en comunidades vegetales. Medio Ambiente 9(1): 140-142.

KNAPP, R. -1984- Sampling methods and taxon analysis in vegetation science. Dr. W Junk Publishers. La Haya.

KREEB, K.H. -1983-Vegetationskunde. E. Ulmer. Stuttgart.

MONTALDO, P. - 1975- Sinecología de las praderas antropogénicas en la provincia de Valdivia, Chile. Agro Sur 3(1): 16-24.

NOVOA, R. y S. VILLASECA -1989- Mapa agroclimático de Chile. INIA. Ministerio de Agricultura. Santiago.

NUÑEZ, L. -1987- Area mínima y su aplicación en asociaciones vegetales del centro-sur de Chile. Tesis de grado (inéd.). Escuela de Ciencias. Univ. Austral de Chile. Valdivia.

OBERDORFER, E. -1960- Pflanzensoziologische Studien in Chile - Ein Vergleich mit Europa. Flora et Vegetatio Mundi 2: 1-208.

ORLOCI, L. -1975-Multivariate analysis in vegetation research. Dr. W. Junk Publishers. La Haya.

RAMÍREZ, C. y R. WESTERMEIER -1976- Estudio de la vegetación espontánea del Jardín Botánico de la Universidad Austral de Chile (Valdivia) como ejemplo de tabulación fitosociológica. Agro Sur 4: 93-105.

RAMIÍREZ, C., C. SAN MARTIN y M.L. KEIM 1996- La vegetación espontánea del antiguo basural de Ovejería (Osorno, Chile). Medio Ambiente 13(1): 42-57.

RAMÍREZ, C., C. SAN MARTÍN y P. OJEDA 1997- Estructura florística, sintaxonomía y dinámica vegetacional del matorral de PicaPica (Cautín, Chile). Meridiano (En prensa).

RAMIREZ, C., C. SAN MARTIN y J. SAN MARTIN -1996- Estructura florística de los bosques pantanosos de Chile central. En: J. Armesto, M.T. Kalin-Arroyo y C. Villagrán (Ed.) Ecología del bosque nativo de Chile. Edit. Universitaria. Santiago. 215-234.

RAMÍREZ, C., V. FINOT, C. SAN MARTÍN y A. ELLIES -1991- El valor indicador ecológico de las malezas del centro-sur de Chile. Agro Sur 19: $94-116$. 
RAMÍREZ, C., E. HAUENSTEIN, D. CONTRERAS y J. SAN MARTÍN -1988- Degradación de la vegetación en la depresión intermedia de la Araucanía, Chile. Agro Sur 16(1): 1-14.

RAMÍREZ, C., C. SAN MARTÍN, A. ELLIES y R. MAC DONALD. 1994. Cambios florísticos desde el bosque nativo a comunidades antropogénicas sometidas a diferentes manejos agropecuarios en un suelo trumao (Valdivia, Chile). Agro Sur 22(1): 57-72.

RAMÍREZ, C., J. SAN MARTÍN, E. HAUENSTEIN y D. CONTRERAS -1989- Estudio fitosociológico de la vegetación de Rucamanque (Cautín, Chile). Studia Botanica 8: 95-115.

SAIZ, F. -1980- Experiencia en el uso de criterios de similitud en el estudio de comunidades. Arch. Biol. Med. Exp. 13: 387-402.

SAN MARTÍN, C., C. RAMÍREZ \& P. OJEDA 1997- Chilean vernal pools: I. Floristic composition.Rhodora (En prensa).

SAN MARTÍN, C. -1992- Flora, vegetación y dinámica vegetacional de la Laguna Santo Domingo (Valdivia, Chile). Tesis (inéd.)Escuela de Graduados. Univ. Austral de Chile. Valdivia.

SAN MARTÍN, C., G. RODRÍGUEZ y C. RAMÍREZ -1991- Origen de la vegetación actual del sector Rapaco-Pichirropulli (Valdivia, Chile). Actas II Congreso Internacional Gestión en Recursos Naturales Valdivia, 2: 456-468.

SAN MARTÍN, C., D. CONTRERAS, J. SAN MARTÍN y C. RAMÍREZ -1992- Vegetación de las marismas del Centro-Sur de Chile. Revista Chilena de Historia Natural 65: 327-342.
SAN MARTÍN, C., R. MEDINA, P. OJEDA y C. RAMÍREZ -1993- La biodiversidad vegetacional del santuario de la naturaleza «Río Cruces» (Valdivia, Chile). Acta Bot. Malacitana 18: 259-279.

SAN MARTÍN, C., C. RAMÍREZ, H. FIGUEROA y N. OJEDA -1991- Estudio sinecológico del bosque de roble-laurel-lingue del centro-sur de Chile. Bosque 12(2): 11-27.

Aceptado para su publicación en Abril de 1998

Dirección de los autores. Instituto de Botánica, Universidad Austral de Chile, Casilla 567, Valdivia, Chile. 